\title{
Theoretical study of mixing in liquid clouds - Part 1: Classical concepts
}

\author{
Alexei Korolev ${ }^{1}$, Alex Khain ${ }^{2}$, Mark Pinsky ${ }^{2}$, and Jeffrey French ${ }^{3}$ \\ ${ }^{1}$ Environment and Climate Change Canada, Cloud Physics and Severe Weather Section, Toronto, Canada \\ ${ }^{2}$ Department of Atmospheric Sciences, the Hebrew University of Jerusalem, Jerusalem, Israel \\ ${ }^{3}$ University of Wyoming, Laramie, WY, USA
}

Correspondence to: Alexei Korolev (alexei.korolev@canada.ca)

Received: 17 August 2015 - Published in Atmos. Chem. Phys. Discuss.: 4 November 2015

Revised: 21 May 2016 - Accepted: 26 May 2016 - Published: 28 July 2016

\begin{abstract}
The present study considers final stages of incloud mixing in the framework of classical concept of homogeneous and extreme inhomogeneous mixing. Simple analytical relationships between basic microphysical parameters were obtained for homogeneous and extreme inhomogeneous mixing based on the adiabatic consideration. It was demonstrated that during homogeneous mixing the functional relationships between the moments of the droplets size distribution hold only during the primary stage of mixing. Subsequent random mixing between already mixed parcels and undiluted cloud parcels breaks these relationships. However, during extreme inhomogeneous mixing the functional relationships between the microphysical parameters hold both for primary and subsequent mixing. The obtained relationships can be used to identify the type of mixing from in situ observations. The effectiveness of the developed method was demonstrated using in situ data collected in convective clouds. It was found that for the specific set of in situ measurements the interaction between cloudy and entrained environments was dominated by extreme inhomogeneous mixing.
\end{abstract}

\section{Introduction}

Turbulent mixing is an important non-adiabatic process in the atmosphere that to a large extent determines spatial gradients of many thermodynamic (e.g., temperature, humidity) and cloud microphysical parameters (e.g., hydrometeor concentrations, extinction coefficient, condensed water content) and as such, needs to be properly described in numerical sim- ulations of clouds and weather predictions. Entrainment and mixing occurs during the entire lifetime of a cloud and is active not only near cloud edges, but is important throughout the whole cloud volume. Mixing of cloudy and entrained air results in changes to the shape of the droplet size distribution through partial droplet evaporation and can also lead to changes in droplet concentration through complete evaporation of some fraction of droplets and dilution. The shape of the droplet size distribution plays a key role in the initiation of precipitation and radiative properties of clouds.

The treatment of mixing in numerical simulations of clouds and precipitation formation remains a challenging problem. Besides the issues related to the way to describe mixing in numerical schemes, there is a fundamental problem of identifying a scenario or path that mixing events should follow. Through the pioneering works of Latham and Reed (1977) and Baker et al. (1980), two explicitly alternative scenarios of mixing were identified. In the first scenario turbulent mixing rapidly stirs the environment homogenizing the fields of temperature and humidity. Following that, all of the droplets undergo partial evaporation under the same conditions. The result of this mixing is a droplet population with reduced sizes, but a total number of droplets remain unchanged. This type of mixing is referred to as homogeneous. In the second scenario mixing occurs more slowly, such that the population of droplets experiences a different amount of sub-saturation. Some number of droplets completely evaporates, while others experience no evaporation until the entirety of the entrained air becomes saturated. Following that, turbulence mixes the rest of the droplets with the saturated, but droplet-free environment. During this type of mixing 
the size of droplets remains unchanged; however, their total number is reduced. This type of mixing is called extreme inhomogeneous. The intermediate case when some fraction of droplets evaporates partially, another fraction evaporates completely, and a third fraction remains unchanged, which in some works is referred to as inhomogeneous (e.g., Baker et al., 1980).

The conditions for homogeneous and extreme inhomogeneous mixing and their effects on precipitation formation have been debated in cloud physics over 40 years. There are a number of numerical simulations and theoretical efforts on studying different aspects of mixing and its effect on cloud microphysics (e.g., Baker and Latham, 1979, 1982; Jensen and Baker, 1989; Su et al., 1998; Lasher-Trapp et al., 2005; Jeffery, 2007; Andrejczuk et al., 2009; Kumar et al., 2013; Jarecka et al., 2013; Lu et al., 2011, 2014; Tolle and Krueger, 2014, and many others). A comprehensive review of the works on the effect of turbulence and mixing on cloud droplet formation can be found in Devenish et al. (2012).

A number of studies were dedicated to identifying a type of mixing based on in situ observations. Most of the previous observations provided evidence supporting inhomogeneous mixing (e.g., Hill and Choularton, 1985; Paluch, 1986; Bower and Choularton, 1988; Blyth and Latham, 1991; Gerber et al., 2008; Lu et al., 2011; Beals et al., 2015). However, works of Jensen and Baker (1989), Paluch and Baumgardner (1989), Burnet and Brenguier (2007), Lehmann et al. (2009), Lu et al. (2011) suggested an occurrence of homogeneous mixing. So, at the moment it appears that both types of mixing may occur in liquid clouds. However, the environmental conditions governing one or the other type of mixing remain not well understood.

Early experimental works on identifying the type of mixing from in situ observations were based on the analysis of spatial variability of the shapes of individual droplet size distributions (e.g., Paluch and Knight, 1984; Paluch, 1986; Bower and Choularton, 1988). The effectiveness of this method involving the analysis of a large number of individual size spectra turned out to be quite low. Another technique utilized expected functional relationships between droplet concentration $(N)$ and droplet diameter $(D)$ specific to each type of mixing. Thus, during extreme inhomogeneous mixing the droplet size is expected to remain unchanged, whereas the concentration will vary. During homogeneous mixing the droplet size and concentration in cloud will be related to each other in a certain way, depending on the mixing fraction and the humidity of the entrained air. This fact was used in observational studies for identifying the type of mixing from "mixing diagrams" that related $N$ and $D_{\mathrm{v}}$ for different regimes of mixing (e.g., Burnet and Brenguier, 2007; Gerber et al., 2008; Lehmann et al., 2009).

The use of mixing diagrams to some extent facilitated identification of type of mixing. However, in many cases scatter in the relationships between $N$ vs. $D_{\mathrm{v}}$ was too large, hindering identification of the type of mixing (Burnet and
Brenguier, 2007). To resolve this problem many researchers used other complementary measurements supporting identification of the type of mixing (e.g., Gerber et al., 2008; Lehmann et al., 2009).

Besides the effect on $N$ and $D_{\mathrm{v}}$, the type of mixing is anticipated to manifest itself in relationships between other moments of the droplet size distribution, $f(D)$. Such relationships may provide insight into the mixing process and identify type of mixing. With the exception of the work by Hill and Choularton (1985), who correlated concentration and liquid water content, there have been few attempts to use any other microphysical parameters for identification of type of mixing.

In order to fill this gap, this study presents a theoretical analysis of relationships between different moments of $f(D)$ within the framework of homogeneous and extreme inhomogeneous mixing. The analysis is focused on the first four moments of $f(D)$ corresponding to the droplet concentration $N$ (0th moment), integral diameter $N \bar{D}$ (1st moment), extinction coefficient $\beta$ (2nd moment), liquid water mixing ratio $q$ (3rd moment) and mean volume diameter $D_{\mathrm{v}}$ (mixed 3rd and 0th moment). It is shown that the newly obtained relationships between the moments provide a more robust identification of type of mixing from in situ measurements as compared to conventional $N-D_{\mathrm{v}}^{3}$ relationships used in mixing diagrams. Relationships between moments may be useful for parameterization of mixing in numerical simulations of clouds and climate and for the interpretations of remotesensing measurements.

This paper constitutes the first in a series of three papers. It considers the final stage of mixing based on the formal definitions of homogeneous and extreme inhomogeneous mixing. These two types of mixing present two extreme regimes of mixing. The following two papers provide a detailed analysis of the time-dependent processes during homogeneous (Pinsky et al., 2016a) and inhomogeneous (Pinsky et al., 2016b) mixing where non-extreme regimes are considered as well.

This paper is arranged in the following way. Section 2 presents analysis of the analytical relationship between $N$, $N \bar{D}, \beta, q, D_{\mathrm{v}}$ and mixing fraction $\mu$ for the cases of homogeneous and extreme inhomogeneous mixing. In Sect. 3 the obtained analytical relationships are compared with the numerical simulations of $N, \beta, q, D_{\mathrm{v}}$ formed at the final stage of mixing. Section 4 presents results of simulation of progressive mixing and its effect of the relationships between moments. Examples of relationship between $N, \beta, q$ and $D_{\mathrm{v}}$ from in situ observations are provided in Sect. 5. The discussion and concluding remarks are presented in Sects. 6 and 7. 

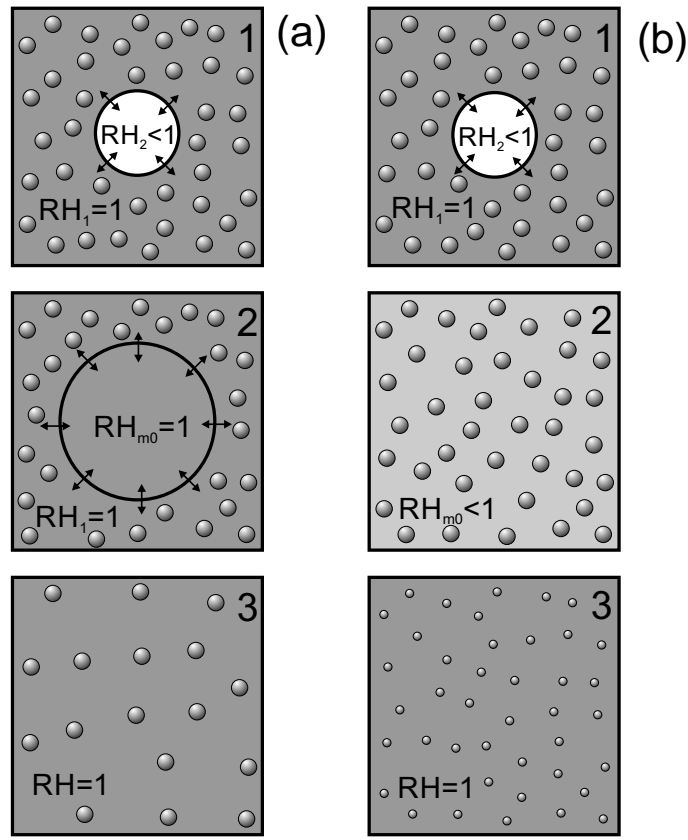

Figure 1. Classical conceptual diagram of (a) inhomogeneous and (b) homogeneous mixing. 1 - initial state; 2 - mixing state; 3 - final state.

\section{Effect of mixing on microphysical variables}

\subsection{Phenomenological consideration}

The conceptual diagrams of homogeneous and extreme inhomogeneous mixing are shown in Fig. 1. During the first stage of extreme inhomogeneous mixing the subsaturated parcel is engulfed into the cloudy environment (Fig. 1a1). Then, the droplets at the interface of the sub-saturated parcel and the cloud environment undergo complete evaporation until the air within the engulfed volume reaches saturation (Fig. 1a2). After that the saturated but droplet-free parcel mixes with the rest of the cloud environment (Fig. 1a3). The result of inhomogeneous mixing is that the cloud parcel has reduced droplet concentration and the droplet sizes remain unchanged.

In the case of homogeneous mixing after entraining into a cloud (Fig. 1b1), the subsaturated parcel "instantly" mixes up with its cloud environment (Fig. 1b2) leading to undersaturation of the total volume. Then, all droplets throughout the mixed volume undergo simultaneous evaporation until the equilibrium state is reached. The result of homogeneous mixing is a cloud volume with reduced concentration of droplets and droplets with reduced sizes (Fig. 1b3).

Based on mass and energy conservation the final state of the bulk parameters (i.e., liquid water mixing fraction, humidity, temperature, etc.) is the same for both types of mixing. However, in the case of extreme inhomogeneous mixing saturation is reached through complete evaporation of some fraction of droplets, and their sizes remain constant. Whereas in case of homogeneous mixing saturation is reached through a uniform evaporation of droplets, and the total number of droplets remains unchanged. It should be noted that in both cases the droplet concentration decreases due to dilution by the mixed droplet-free sub-saturated parcel.

The following discussion will be specifically focused on the microphysical properties formed at the final stage of the homogeneous and extreme inhomogeneous mixing. The processes occurring during mixing state (i.e., transition a1 $\rightarrow$ a2 and $b 1 \rightarrow$ b2 in Fig. 1) remain outside the frame of this work. Following the formalism of homogeneous and extreme inhomogeneous mixing, the process of mixing reaches the final stage when (1) the entrained and cloud environment are mixed up and the spatial gradients of the microphysical $(N$, $\beta, q$, etc.) and environmental $(T, S, e$, etc.) parameters approach zero; (2) the diffusional process related to droplet evaporation comes into equilibrium. The second condition is completed when (a) the environment reaches saturation state, or (b) the entire population of droplets is completely evaporated, if the entrained air is sufficiently dry.

The above description of homogeneous and extreme inhomogeneous mixing is highly idealized. Actual in-cloud mixing does not occur as a sequence of discrete events (Fig. 1) that individually come to equilibrium only to be followed by the next discrete mixing events. But rather it is occurring continuously on a cascade of different spatial and timescales. Broadwell and Breidenthal (1982) summarized the experimental evidence and proposed the following description of mixing in turbulent shear layers. Mixing takes place in a series of events. Two shear layers exchange mass by engulfing parcels from an opposite layer into localized zones. The initially large-scale filaments of the two gases break down towards smaller scales due to the action of turbulence. The turbulence stretches the interface between the gases and enhances the molecular diffusion across the increasing surface. The actual mixing of the engulfed volume is a molecular diffusion process that is most effective after the break down volumes reduce to the Kolmogorov viscosity scale. It is anticipated that the reaction of the ensemble of droplets is a combination of homogeneous and inhomogeneous mixing with domination of one type of mixing over the other depending on the characteristic spatial and timescales of the environment determined by turbulence, cloud microphysics, state parameters and stage of mixing.

\subsection{Methodology}

The foregoing discussion will be focused on mixing between saturated cloud parcels and out-of-cloud sub-saturated air. The cloud parcel contains droplets with average diameter $\bar{D}_{1}$, liquid mixing ratio $q_{1}$ and number concentration $N_{1}$. The initial temperature in the cloud parcel is $T_{1}$, relative humidity $\mathrm{RH}_{1}=1$, where $\mathrm{RH}=e / e_{s}(T)$ (the explanation of variable notations is provided in Table 1). The second parcel is 
Table 1. List of symbols.

\begin{tabular}{|c|c|c|}
\hline Symbol & Description & Units \\
\hline$A_{2}$ & $\frac{p R_{\mathrm{V}}}{e_{s} R_{\mathrm{a}}}+\frac{L^{2}}{c_{p \mathrm{a}} R_{\mathrm{v}} T^{2}}$ & - \\
\hline$a$ & $\frac{e_{S} R_{\mathrm{a}} L^{2}}{p c_{p \mathrm{a}} R_{\mathrm{v}}^{2} T^{2}}$ & - \\
\hline$b$ & $\frac{c_{p \mathrm{a}} R_{\mathrm{V}} T^{2}}{L^{2}}$ & - \\
\hline$c_{p \mathrm{a}}$ & Specific heat capacity of dry air at constant pressure & $\mathrm{J} \mathrm{kg}^{-1} \mathrm{~K}^{-1}$ \\
\hline$c_{p \mathrm{~V}}$ & Specific heat capacity of water vapor at constant pressure & $\mathrm{J} \mathrm{kg}^{-1} \mathrm{~K}^{-1}$ \\
\hline$\stackrel{P}{D}$ & Mean droplet diameter & $\mathrm{m}$ \\
\hline$D_{2}$ & Mean square droplet diameter & $\mathrm{m}$ \\
\hline$D_{\mathrm{v}}$ & Mean volume droplet diameter & $\mathrm{m}$ \\
\hline$e$ & Water vapor pressure & $\mathrm{Nm}^{-2}$ \\
\hline$e_{1}$ & Initial water vapor pressure in the cloud parcel & $\mathrm{N} \mathrm{m}^{-2}$ \\
\hline$e_{2}$ & Initial water vapor pressure in the entrained sub-saturated parcel & $\mathrm{Nm}^{-2}$ \\
\hline$e_{s}$ & Saturation vapor pressure above flat surface of water & $\mathrm{Nm}^{-2}$ \\
\hline$f(D)$ & Size distribution of cloud droplets normalized on unity & $\mathrm{m}^{-1}$ \\
\hline$L$ & Latent heat of vaporization for liquid water & $\mathrm{J} \mathrm{kg}^{-1}$ \\
\hline$M_{n}$ & $n$th moment of the droplet size distribution $\frac{\int_{0}^{\infty} f(D) D^{n} \mathrm{~d} D}{\int_{0}^{\infty} f(D) \mathrm{d} D}$ & $\mathrm{~m}^{n}$ \\
\hline$N$ & Concentration of droplets & $\mathrm{m}^{-3}$ \\
\hline$N_{1}$ & Concentration of droplets before mixing & $m^{-3}$ \\
\hline$p$ & Pressure of moist air & $\mathrm{Nm}^{-2}$ \\
\hline$R_{\mathrm{a}}$ & Specific gas constant of moist air & $\mathrm{J} \mathrm{kg}^{-1} \mathrm{~K}^{-1}$ \\
\hline$R_{\mathrm{V}}$ & Specific gas constant of water vapor & $\mathrm{J} \mathrm{kg}^{-1} \mathrm{~K}^{-1}$ \\
\hline RH & $e / e_{s}$, relative humidity over water (saturation ratio) & - \\
\hline $\mathrm{RH}_{1}$ & Initial relative humidity in the cloud volume $\left(\mathrm{RH}_{1}=1\right)$ & - \\
\hline $\mathrm{RH}_{2}$ & Relative humidity in the entrained sub-saturated parcel & - \\
\hline $\mathrm{RH}_{\mathrm{m} 0}$ & $\begin{array}{l}\text { Relative humidity after instant mixing of cloudy and entrained air, but before droplets evapora- } \\
\text { tion }\end{array}$ & - \\
\hline$q$ & Cloud liquid water mixing ratio (mass of liquid water per $1 \mathrm{~kg}$ of dry air) & - \\
\hline$q_{1}$ & Cloud liquid water mixing ratio before mixing & - \\
\hline$q_{\mathrm{v}}$ & Water vapor mixing ratio (mass of water vapor per $1 \mathrm{~kg}$ of dry air) & - \\
\hline$S$ & $e / e_{S}-1$, supersaturation & - \\
\hline$S_{2}$ & Supersaturation of the dry out-of-cloud air & - \\
\hline$S_{\mathrm{m} 0}$ & $\begin{array}{l}\text { Supersaturation after instant mixing of cloudy and entrained air, but before droplets start evap- } \\
\text { orating }\end{array}$ & - \\
\hline$T$ & Temperature & $\mathrm{K}$ \\
\hline$T_{1}$ & Temperature of the cloud parcel before mixing & $\mathrm{K}$ \\
\hline$T_{2}$ & Temperature of the entrained sub-saturated parcel before mixing & $\mathrm{K}$ \\
\hline$T_{\mathrm{m} 0}$ & Temperature of the parcel after vapor mixing, but before droplet evaporation & $\mathrm{K}$ \\
\hline$\beta$ & Extinction coefficient & $m^{-1}$ \\
\hline$\beta_{1}$ & Extinction coefficient before mixing & $\mathrm{m}^{-1}$ \\
\hline$\delta q_{\mathrm{m}}$ & $\begin{array}{l}\text { Mixing ratio of liquid water required to saturate } 1 \mathrm{~kg} \text { of the cloud volume after instant mixing, } \\
\text { but before droplet evaporation. }\end{array}$ & - \\
\hline$\delta q^{*}$ & Mixing ratio of liquid water required to saturate $1 \mathrm{~kg}$ of the dry out-of-cloud air & - \\
\hline$\mu$ & Cloud fraction of mixing air, $0 \leq \mu \leq 1$ & - \\
\hline$\mu_{\mathrm{cr}}$ & Critical cloud fraction, such that for $\mu \leq \mu_{\mathrm{cr}}$ all droplets evaporate & - \\
\hline$\rho_{\mathrm{a}}$ & Density of the dry air & $\mathrm{kg} \mathrm{m}^{-3}$ \\
\hline$\rho_{\mathrm{W}}$ & Density of liquid water & $\mathrm{kg} \mathrm{m}^{-3}$ \\
\hline$\xi$ & $\begin{array}{l}\text { Coefficient } 0 \leq \xi \leq 1 \text { characterizing proximity of homogeneous mixing to extremely inhomo- } \\
\text { geneous mixing (i.e., when } \xi \rightarrow 0 \text { ). }\end{array}$ & - \\
\hline
\end{tabular}


droplet free $\left(N_{2}=0\right)$, sub-saturated with initial relative humidity $\mathrm{RH}_{2}<1$ and temperature $T_{2}$. The mixing occurs isobarically, i.e., $p=$ const. At the final stage of mixing the temperature and humidity formed in the resulting parcel are $T$ and RH (Appendix A). The process of mixing is completed, when the mixed parcel reaches equilibrium due to the air saturation (i.e., $\mathrm{RH}=1$ ), or due to the complete evaporation of droplets. In the latter case the final humidity is $\mathrm{RH} \leq 1$. The effect of the vertical velocity and vertical travel on final $T, \mathrm{RH}$, and $q$ is not considered here, i.e., vertical velocity $u_{z}=0$.

Without the loss of generality the masses of the cloudy and sub-saturated volumes prior to the mixing are assumed to have a unit masses, i.e., $m_{1}=1$ and $m_{2}=1$. The mixing process will be considered as mixing of $\mu$ fraction of the cloud parcel with $(1-\mu)$ fraction of the second (sub-saturated) parcel. The mixing cloud fraction may vary within the range of $0 \leq \mu \leq 1$. Therefore, the mass of the resulting mixed parcel is equal to $m_{1} \mu+(1-\mu) m_{2}=1$. This approach simplifies the consideration of mixing and allows us to consider all possible proportions of the mixing of two volumes.

\subsection{Effect of mixing on liquid water and temperature}

The mixing ratio of liquid water $q$ formed at the final stage of mixing is determined by the mass of the mixing cloud water $\mu q_{1}$ and amount of evaporated water required to saturate the newly formed mixed volume $\delta q_{\mathrm{m}}$. The mass balance of liquid water for the mixing volume yields

$q=\mu q_{1}-\delta q_{\mathrm{m}}$,

where

$\delta q_{\mathrm{m}}=\frac{c_{p \mathrm{a}} R_{\mathrm{v}} T_{\mathrm{m} 0}^{2}}{L^{2}} \ln \left(\frac{1+\frac{e_{s}\left(T_{\mathrm{m} 0}\right) R_{\mathrm{a}} L^{2}}{p c_{p \mathrm{a}} R_{\mathrm{v}}^{2} T_{\mathrm{m} 0}^{2}}}{1+\mathrm{RH}_{\mathrm{m} 0} \frac{e_{s}\left(T_{\mathrm{m} 0}\right) R_{\mathrm{a}} L^{2}}{p c_{p \mathrm{a}} R_{\mathrm{v}}^{2} T_{\mathrm{m} 0}^{2}}}\right) \cong-\frac{S_{\mathrm{m} 0}}{A_{2}}$

is the mixing ratio of liquid water required to saturate $1 \mathrm{~kg}$ of volume with temperature $T_{\mathrm{m} 0}$ and humidity $\mathrm{RH}_{\mathrm{m} 0}$ (Appendix A); $T_{\mathrm{m} 0}, \mathrm{RH}_{\mathrm{m} 0}$ and $S_{\mathrm{m} 0}$, are the temperature, relative humidity and supersaturation formed in the volume after instantaneous air mixing, but before droplets start evaporating (Appendix A); $e_{s}\left(T_{\mathrm{m} 0}\right)$ is the saturation vapor pressure at temperature $T_{\mathrm{m} 0}$.

Equation (1) is a non-linear function of $\mu$, since $T_{\mathrm{m} 0}, e_{\mathrm{m} 0}$ and thus $\delta q_{\mathrm{m}}$ depend on $\mu$. Equation (1) can be simplified, if $T_{1}=T_{2}$. In this case $T_{\mathrm{m} 0}=T_{1}=T_{2}$, and $e_{s}\left(T_{\mathrm{m} 0}\right)=e_{s}\left(T_{1}\right)=$ $e_{s}\left(T_{2}\right)$. Given that, the expression under logarithm in Eq. (2) can be expanded in series resulting in (Appendix B)

$\delta q_{\mathrm{m}}=(1-\mu) \delta q^{*}$,

where $\delta q^{*}=\frac{c_{p \mathrm{a}} R_{\mathrm{v}} T_{2}^{2}}{L^{2}} \ln \left(\frac{1+\frac{e_{s}\left(T_{2}\right) R_{\mathrm{a}} L^{2}}{p c_{p \mathrm{a}} R_{\mathrm{v}}^{2} T_{2}^{2}}}{1+\mathrm{RH}_{2} \frac{e_{s}\left(T_{2}\right) R_{\mathrm{a}} L^{2}}{p c_{p \mathrm{a}} R_{\mathrm{v}}^{2} T_{2}^{2}}}\right) \cong-\frac{S_{2}}{A_{2}}$

is the mixing ratio of liquid water required to saturate $1 \mathrm{~kg}$ of the entrained dry air with temperature $T_{2}$ and humidity $\mathrm{RH}_{2}$. Substituting Eq. (3) in Eq. (1) gives

$q=\mu q_{1}-(1-\mu) \delta q^{*}$.

The value of $\delta q^{*}$ does not depend on $\mu$, and Eq. (5) is a simple linear function of $\mu$. The comparisons with numerical simulations showed that Eq. (5) provides accuracy within a few percent, when the temperature difference $\left|T_{1}-T_{2}\right|<2^{\circ}$. Although, in many cases $\left|T_{1}-T_{2}\right|$ may vary a wide range reaching $10^{\circ} \mathrm{C}$ or higher, clouds with $\left|T_{1}-T_{2}\right|<2^{\circ}$ are quite common. Therefore, for the sake of simplicity, Eq. (5) and the assumption $T_{1} \approx T_{2}$ will be used in the following consideration of mixing.

It should be noted that Eqs. (1) and (5) are valid for the cases when $\mu>\mu_{\mathrm{cr}}$. Here $\mu_{\mathrm{cr}}$ is critical mixing fraction, which separates partial and complete evaporation of cloud water in the mixing volume (Sect. 2.4). Cases when $\mu \leq \mu_{\mathrm{cr}}$ correspond to complete evaporation of droplets, and $q=0$.

The temperature at the final stage of mixing can be estimated as (Appendix C)

$$
\begin{aligned}
& T=T_{\mathrm{m} 0}-\frac{(1-\mu) \delta q^{*} L}{c_{p \mathrm{a}}}, \text { when } \mu>\mu_{\mathrm{cr}} \\
& T=T_{\mathrm{m} 0}-\frac{\mu q_{1} L}{c_{p \mathrm{a}}} \text { when } \mu \leq \mu_{\mathrm{cr}} .
\end{aligned}
$$

Eqs. (1), (5), (6) were obtained based on mass and energy conservation, and they do not depend on how mixing proceeds. Therefore, Eqs. (1), (5), (6) are valid for both homogeneous and inhomogeneous mixing.

\subsection{Complete evaporation}

As mentioned in Sect. 2.2 the process of mixing is complete only after reaching equilibrium by saturating the mixed volume or by evaporating of all cloud droplets depending on the mixing fraction $\mu$. The critical mixing fraction $\mu_{\mathrm{cr}}$, corresponding to evaporation of all droplets, can be found from Eq. (5) when $q=0$, i.e.,

$\mu_{\mathrm{cr}}=\frac{\delta q^{*}}{q_{1}+\delta q^{*}}$.

Critical mixing fraction separates $\mu$ in two subranges: (a) $1 \geq$ $\mu>\mu_{\mathrm{cr}}$ where $q$ is described by Eqs. (1) or (5) and $\mathrm{RH}_{\mathrm{m}}=$ 1 ; (b) $\mu_{\mathrm{cr}} \geq \mu \geq 0$ where $q=0$ and $\mathrm{RH}_{\mathrm{m}} \leq 1$.

For the general case when $T_{1} \neq T_{2}, \mu_{\mathrm{cr}}$, can be found by solving the non-linear equation

$\mu_{\mathrm{cr}} q_{1}-\delta q_{\mathrm{m}}\left(\mu_{\mathrm{cr}}\right)=0$. 


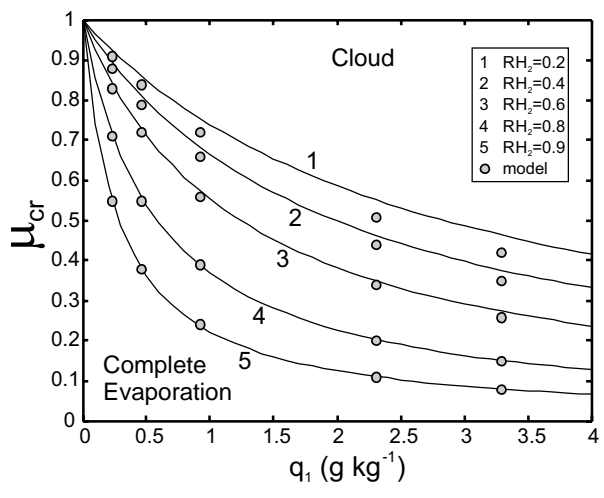

Figure 2. Dependence of critical mixing fraction $\mu_{\mathrm{cr}}$ vs. mixing ratio $q_{0}$ calculated from Eq. (7). Circles indicate modeled points. The calculations were performed for $T=0^{\circ} \mathrm{C}$ and $H=3000 \mathrm{~m}$.

Figure 2 shows comparisons of dependences of $\mu_{\mathrm{cr}}$ vs. $q_{1}$ calculated from Eq. (7) and those deduced from a numerical model (Sect. 3). Critical mixing fraction $\mu_{\mathrm{cr}}$ is also shown by black stars in Fig. 4. The locations of the stars in Fig. 4 coincide well with the locations, where the modeled microphysical moments become zero. The obtained agreement between analytical and modeled $\mu_{\mathrm{cr}}$ in Figs. 2 and 4 validates the developed approach.

\subsection{Extreme inhomogeneous mixing}

Within the framework of extreme inhomogeneous mixing some fraction of droplets undergo complete evaporation, whereas the rest of the droplets remain unchanged. Therefore, such a process results in scaling the droplet size distribution $f(D)$, i.e.,

$f(D)=k f_{1}(D)$,

where $k$ is some coefficient dependent on $\mu$ and the initial environmental parameters of the mixing volumes, $f_{1}(D)$ is the droplet size distribution before mixing. Equation (9) yields relationships between pairs $n$th and $m$ th moments

$\frac{M_{n}}{M_{n 1}}=\frac{M_{m}}{M_{m 1}}$,

where $M_{n}$ is the $n$th moment of $f(D)$. Therefore, it is anticipated that for extreme inhomogeneous mixing droplet number concentration $N$ (0th moment), extinction coefficient $\beta$ (2nd moment), liquid water mixing ratio $q$ (3rd moment), along with other moments, will correlate with each other, i.e.,

$\frac{N}{N_{1}}=\frac{\beta}{\beta_{1}}=\frac{q}{q_{1}}$.

One of the consequences of Eqs. (9)-(10) is that the characteristic droplet sizes $\bar{D}, D_{2}, D_{\mathrm{v}}, D_{\text {eff }}$ will remain constant during inhomogeneous mixing.
For the case $T_{1}=T_{2}$ and $\mu>\mu_{\text {cr }}$ Eqs. (5) and (11) yield the dependence of $N$ vs. $\mu$

$N=N_{1}\left(\mu-\frac{(1-\mu) \delta q^{*}}{q_{1}}\right)$

$\beta=\beta_{1}\left(\mu-\frac{(1-\mu) \delta q^{*}}{q_{1}}\right)$.

For a general case when $T_{1} \neq T_{2}$ the term $(1-\mu) \delta q^{*}$ in Eqs. (12) and (13) should be replaced by $\delta q_{\mathrm{m}}(\mu)$ (Eq. 2).

\subsection{Homogeneous mixing}

For homogeneous mixing, when $\mu>\mu_{\mathrm{cr}}$, the droplet number concentration changes only due to dilution by the entrained air, i.e.,

$\frac{N}{N_{1}}=\mu$.

Assuming $T_{1}=T_{2}$, and substituting Eq. (5) in Eq. (14) yields

$\frac{N}{N_{1}}=\frac{q+\delta q^{*}}{q_{1}+\delta q^{*}}$.

Following Eq. (15) $N$ and $q$ are linearly related for homogeneous mixing. However, no linear relationships exist between other moments. Thus, substituting the definition of the liquid water mixing ratio $q=\pi \rho_{\mathrm{w}} N D_{\mathrm{v}}^{3} / 6 \rho_{\mathrm{a}}$ in Eq. (15) yields the relationship between mean volume droplet size, concentration and liquid water mixing fraction

$$
\begin{aligned}
& \frac{D_{\mathrm{v}}^{3}}{D_{\mathrm{v} 1}^{3}}=1+\left(1-\frac{N_{1}}{N}\right) \frac{\delta q^{*}}{q_{1}} \\
& \frac{D_{\mathrm{v}}^{3}}{D_{\mathrm{v} 1}^{3}}=\frac{q}{q_{1}}\left(\frac{q_{1}+\delta q^{*}}{q+\delta q^{*}}\right) .
\end{aligned}
$$

In a similar way the relationship between the extinction coefficient $\beta=Q \pi N D_{2}^{2} / 4, N$ and $q$ can be written as

$$
\begin{aligned}
\frac{\beta}{\beta_{1}} & =\frac{N}{N_{1}}\left(1+\left(1-\frac{N_{1}}{N}\right) \frac{\delta q^{*}}{q_{1}}\right)^{2 / 3} \\
\frac{\beta}{\beta_{1}} & =\left(\frac{q}{q_{1}}\right)^{2 / 3}\left(\frac{q+\delta q^{*}}{q_{1}+\delta q^{*}}\right)^{1 / 3} .
\end{aligned}
$$

In Eqs. (17a) and (17b) it is assumed that $D_{2} \approx D_{\mathrm{v}}$.

Substituting in Eq. (16) the expression for the time of phase relaxation $\tau_{p}=1 / b N \bar{D}$ (e.g., Squires, 1952; Korolev and Mazin, 2003) and assuming $\bar{D} \approx D_{\mathrm{v}}$ yields

$$
\frac{\tau}{\tau_{1}}=\frac{N_{1}}{N}\left(1+\left(1-\frac{N_{1}}{N}\right) \frac{\delta q^{*}}{q_{1}}\right)^{-1 / 3} .
$$

For the cases when the temperature difference $\left|T_{1}-T_{2}\right|$ exceeds a few degrees, the effect of $\mu$ on $T_{\mathrm{m}}$ and $S_{\mathrm{m}}$ should be taken into consideration in the calculations of evaporated 
water. For such cases $\delta q_{\mathrm{m}}$ (Eq. 2) should be used instead of $\delta q^{*}$. Using Eq. (14) $\delta q_{\mathrm{m}}$ can be presented as a function of $\frac{N}{N_{1}}$, i.e., $\delta q_{\mathrm{m}}(\mu)=\delta q_{\mathrm{m}}\left(\frac{N}{N_{1}}\right)$. Replacing Eq. (5) by Eq. (1) in the above consideration, the equations Eqs. (15)-(18) can be rewritten as

$$
\begin{aligned}
\frac{N}{N_{1}} & =\frac{q+\delta q_{\mathrm{m}}\left(\frac{N}{N_{1}}\right)}{q_{1}} \\
\frac{D_{\mathrm{v}}^{3}}{D_{\mathrm{v} 1}^{3}} & =1-\frac{\delta q_{\mathrm{m}}\left(\frac{N}{N_{1}}\right)}{q_{1}} \frac{N_{1}}{N}=\frac{q}{q+\delta q_{\mathrm{m}}\left(\frac{q}{q_{1}}\right)} \\
\frac{\beta}{\beta_{1}} & =\frac{N}{N_{1}}\left(1-\frac{\delta q_{\mathrm{m}}\left(\frac{N}{N_{1}}\right)}{q_{1}} \frac{N}{N_{1}}\right)^{2 / 3} \\
& =\frac{q^{2 / 3}\left(q+\delta q_{\mathrm{m}}\left(\frac{\beta}{\beta_{1}}\right)\right)}{q_{1}} \\
\frac{\tau_{p}}{\tau_{p 1}} & =\frac{N_{1}}{N}\left(1-\frac{\delta q_{\mathrm{m}}\left(\frac{N}{N_{0}}\right)}{q_{1}} \frac{N_{1}}{N}\right)^{-1 / 3} .
\end{aligned}
$$

Equations (19)-(22) can be solved numerically.

\subsection{Degenerate case}

Following Eq. (5), if

$$
\frac{(1-\mu)}{\mu} \frac{\delta q^{*}}{q_{1}} \ll 1
$$

then $q_{1} \geq q \gg \delta q^{*}$. If the condition in Eq. (23) is satisfied, then the terms associated with $\delta q^{*}$ in Eqs. (15)-(18) can be neglected. This results in correlation of all moments, i.e., $N / N_{1}=\beta / \beta_{1}=q / q_{1}$ (compare with Eq. 11). This corresponds to the degenerate case, when the difference between the homogeneous and inhomogeneous mixing vanishes. Thus, the dimensionless parameter $\xi=\frac{1-\mu}{\mu} \frac{\delta q^{*}}{q_{1}}$ in Eq. (23) can be used for characterization of proximity of the homogeneous mixing moments to those formed during extreme inhomogeneous mixing.

The range of $\mu$ in $\xi$ is limited by $\mu_{\mathrm{cr}}<\mu \leq 1$, so that $0<$ $\frac{1-\mu}{\mu} \leq \frac{q_{1}}{\delta q^{*}}$. This gives the range of changes of $\xi$, i.e., $0 \leq \xi \leq$ 1 for the mixing without complete evaporation of all droplets. The degenerate case corresponds to $\xi \rightarrow 0$, whereas $\xi \rightarrow 1$ corresponds to a maximum difference of the moments for homogeneous and extreme inhomogeneous mixing.

As follows from Eqs. (4) and (23) approaching to the degenerate case $(\xi \rightarrow 0)$ occurs, when one of the following conditions or their combination is satisfied: (a) $\mathrm{RH}_{2} \rightarrow 1$; (b) $e_{s}(T) \rightarrow 0$ at low temperatures; (c) $q_{1} \gg \delta q^{*}$; (d) $\mu \rightarrow 1$. The effect of RH, $T, q_{1}$ and $\mu$ on mixing will be demonstrated in Sect. 3.

Figure 3 shows dependence of $\xi$ vs. $\mu$. The grey area in Fig. 3 indicates the region where identification of type of

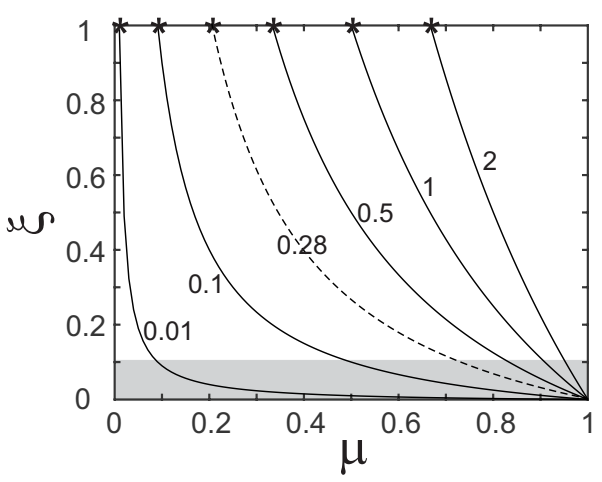

Figure 3. Dependence of $\xi$ vs. $\mu$. Numbers are the dimensionless ratios $\delta q^{*} / q_{1}$. Critical mixing ratios $\mu_{\mathrm{cr}}$ are indicated by stars. Grey color indicates the area where the moments of homogeneous and extreme inhomogeneous mixing may not be segregated from in situ measurements. Dashed line was calculated for the cloud in Figs. 1314.

mixing from in situ measurements (Sect. 5) may be hindered due to proximity of the moments for homogeneous and extreme inhomogeneous mixing. Thus for $\delta q^{*} / q_{1}=0.01$ identification of type of mixing is ambiguous for nearly the entire range of $\mu$.

For the general case, when $T_{1} \neq T_{2}$, it should be $\xi=$ $\frac{\left|\delta q_{\mathrm{m}}(\mu)\right|}{\mu q_{1}}$. An absolute value $\left|\delta q_{\mathrm{m}}(\mu)\right|$ should be used in $\xi$ since $\delta q_{\mathrm{m}}(\mu)$ can be negative (Appendix A, Fig. A1) if mixing results in supersaturation Sect. 3.4).

The coefficient $\xi$ may be useful for identification type of mixing from in situ observations.

In conclusion of Sect. 2 it is worth noting, that for both homogeneous and extremely inhomogeneous mixing most of the relationships between moments include the ratio $\frac{\delta q^{*}}{q_{1}}$. As follows from Eq. (A9) (in Appendix A) $\frac{\delta q^{*}}{q_{1}} \cong \frac{S_{2}}{A_{2} q_{1}}$, which is equal to the so-called "potential evaporation parameter" $R$ (Pinsky et al., 2016a, b). The potential evaporation parameter $R$ plays an important role in determining scenarios of droplet evaporation in a turbulent environment.

\section{Comparisons with numerical simulations}

Numerical simulations were performed to examine accuracy and limitations of the analytical expressions in Sect. 2 and to conduct a sensitivity test to environmental and cloud parameters. The simulations have been performed with the help of a parcel model similar to that in Korolev (1995). The ensemble of droplets in the simulation was assumed to be monodisperse. For the case of extreme inhomogeneous mixing the amount of evaporated water $\Delta q$ required to saturate the mixed volume was calculated first. If $\Delta q<\mu q_{1}$, then the concentration of evaporated droplets was calculated as $N_{\text {ev }}=\frac{\Delta q}{m_{\mathrm{d}}} \rho_{\mathrm{a}}$, where $m_{\mathrm{d}}=\pi \rho_{\mathrm{w}} D^{3} / 6$. Then, the concentration of the remaining droplets $N=N_{1}-N_{\mathrm{ev}}$ was recalculated 
based on the calculation on the volume formed after mixing. If $\Delta q \geq \mu q_{1}$, then all droplets evaporate, and $N=0$.

For the case of homogeneous mixing in the first step the engulfed parcel instantly mixes with the cloud parcel resulting in a new humidity $\mathrm{RH}_{\mathrm{m} 0}$, temperature $T_{\mathrm{m} 0}$ and volume $V_{\mathrm{m} 0}$. After that the droplets start evaporating until either their complete evaporation or saturation over liquid is reached. The calculations are stopped when, either $D<0.2 \mu \mathrm{m}$ or $\left(e_{s}-e\right) / e_{s}<0.001$, respectively.

\subsection{Effect of mixing fraction}

Figure 4 shows the results of the simulation of different moments and state parameters vs. $\mu$. The calculations were performed for different relative humidity of the entrained parcel $\mathrm{RH}_{2}=0.2,0.5,0.8$ and 0.95. As seen from Fig. 4 for the case of homogeneous mixing only $N$ and $q$ are linearly related with $\mu$, the rest of the variables have non-linear dependences on $\mu$. For the case of extreme inhomogeneous mixing all $f(D)$ moments and droplet sizes linearly depend on $\mu$. Note, for $\mu \leq \mu_{\mathrm{cr}}$ all moments are equal to zero.

Since the amount of the evaporated liquid water does not depend on the type of mixing, the dependences of $q(\mu)$ are the same for both homogeneous and inhomogeneous mixing (Fig. 4a). The type of mixing has the most pronounced effect on the droplet concentration (Fig. 4b) and droplet sizes (Fig. 4e).

Figure $4(\mathrm{~g})$ shows the dependences $\mathrm{RH}_{\mathrm{m} 0}$ and $\mathrm{RH}$ vs. $\mu$. Here $\mathrm{RH}_{\mathrm{m} 0}$ is the relative humidity at the initial stage of homogeneous mixing before droplets start evaporating (Fig. 1b2). Figure $4 \mathrm{~h}$ presents comparisons of modeled $T(\mu)$ and those calculated from Eqs. (6a), (6b) and (C4). The independence of $q(\mu), \mathrm{RH}(\mu)$ and $T(\mu)$ on type of mixing (Fig. $4 \mathrm{a}, \mathrm{g}, \mathrm{h}$ ) is the consequence of the mass and energy conservation, which are not contingent on type of mixing.

\subsection{Effect of humidity of entrained air}

The diagrams in Fig. 5a-c show the dependences of normalized $\beta, q$ and $D_{\mathrm{v}}$ vs. $N / N_{1}$ calculated from numerical simulations and analytical equations from Sect. 2. The calculations were performed for different humidity of the entrained air $\mathrm{RH}_{2}$. As seen from Fig. 5a-c, the normalized dependences for homogeneous mixing $q(N), \beta(N)$ and $D_{\mathrm{v}}(N)$ tend to approach the line of extreme inhomogeneous mixing when relative humidity $\mathrm{RH}_{2}$ approaches 1 . This is consistent with the degenerate case, when $\xi \rightarrow 0$ (Sect. 2.7). In this case droplets behave as a passive admixture, and they do not interact with the environment.

\subsection{Effect of liquid water mixing ratio}

Figure $5 \mathrm{~d}-\mathrm{f}$ demonstrate the sensitivity of $q(N), \beta(N)$ and $D_{\mathrm{v}}(N)$ to liquid water mixing ratio $q_{1}$. It is seen that the increase of $q_{1}$ results in $q(N), \beta(N)$ and $D_{\mathrm{v}}(N)$ (calculated for homogeneous mixing) approaching towards $q(N)$,
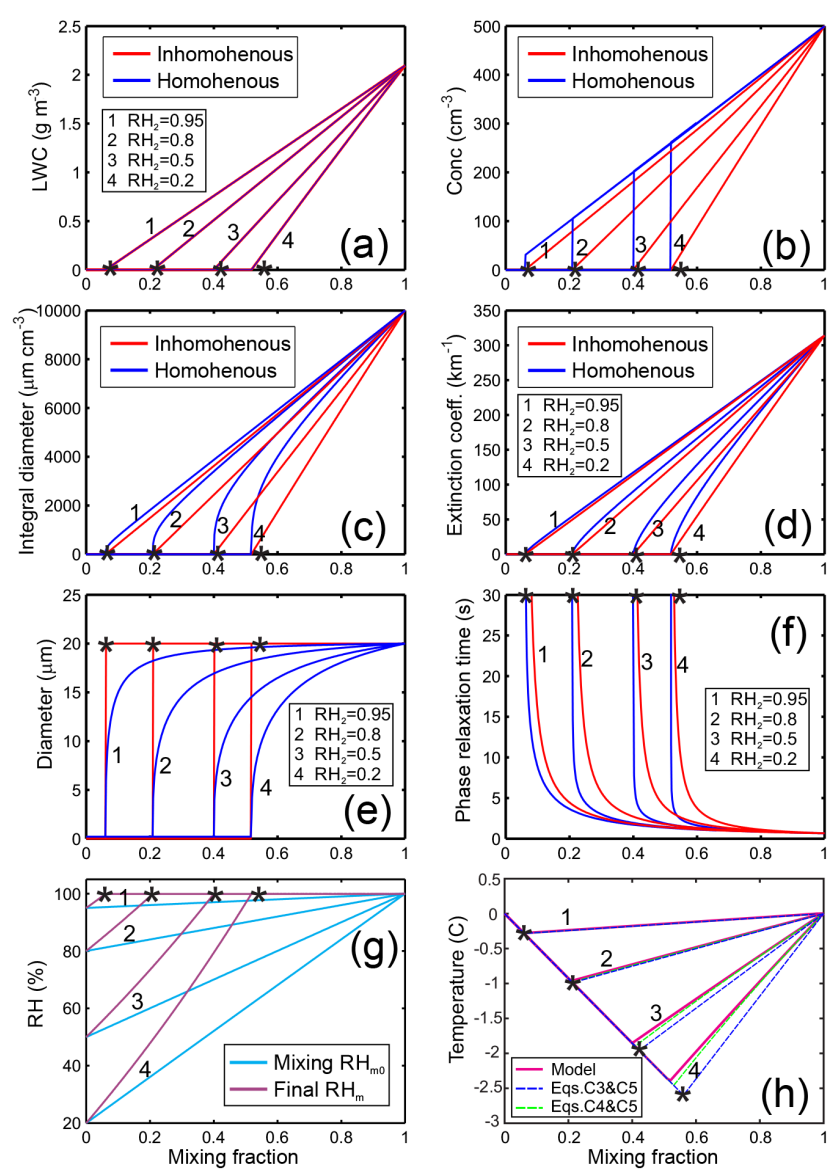

Figure 4. Simulation of (a) liquid water mixing ratio, (b) droplet number concentration, (c) integral droplet diameter, (d) extinction coefficient, (e) mean volume diameter, (f) time of phase relaxation, (g) relative humidity in the mixed volume before droplet evaporation $\mathrm{RH}_{\mathrm{m} 0}$ and at the equilibrium state $\mathrm{RH}_{\mathrm{m}}$, (h) final temperature $T_{\mathrm{m} 0}$ vs. ratio of mixing $\mu$ formed after homogeneous and extreme inhomogeneous mixing between dry and cloudy parcel with monodisperse droplets. Black stars indicate critical mixing fraction $\mu_{\mathrm{cr}}$ calculated from Eq. (7). The calculations were performed for $\mathrm{RH}_{2}=0.2,0.5,0.8,0.95 ; D_{1}=20 \mu \mathrm{m}, N_{1}=500 \mathrm{~cm}^{-3} ; T_{1}=$ $T_{2}=0{ }^{\circ} \mathrm{C} ; H=1000 \mathrm{~m}$.

$\beta(N)$ and $D_{\mathrm{v}}(N)$ for the inhomogeneous mixing. In other words, the sensitivity of the microphysical parameters to the type of mixing increases with the decrease of $q_{1}$. From a practical viewpoint it means that from in situ observations the difference between homogeneous and inhomogeneous mixing is anticipated to be more pronounced for the cases with a relatively low liquid water mixing ratio (e.g., $q_{1}<1 \mathrm{~g} \mathrm{~kg}^{-1}$ ). Such behavior is consistent with the consideration in Sect. 2.7.

\subsection{Effect of temperature, case $T_{1}=T_{2}$}

Figure 5g-i shows the effect of temperature on the normalized $q(N), \beta(N)$ and $D_{\mathrm{v}}(N)$ for $T_{1}=T_{2}$. Figure $5 \mathrm{~g}-\mathrm{i}$ indi- 

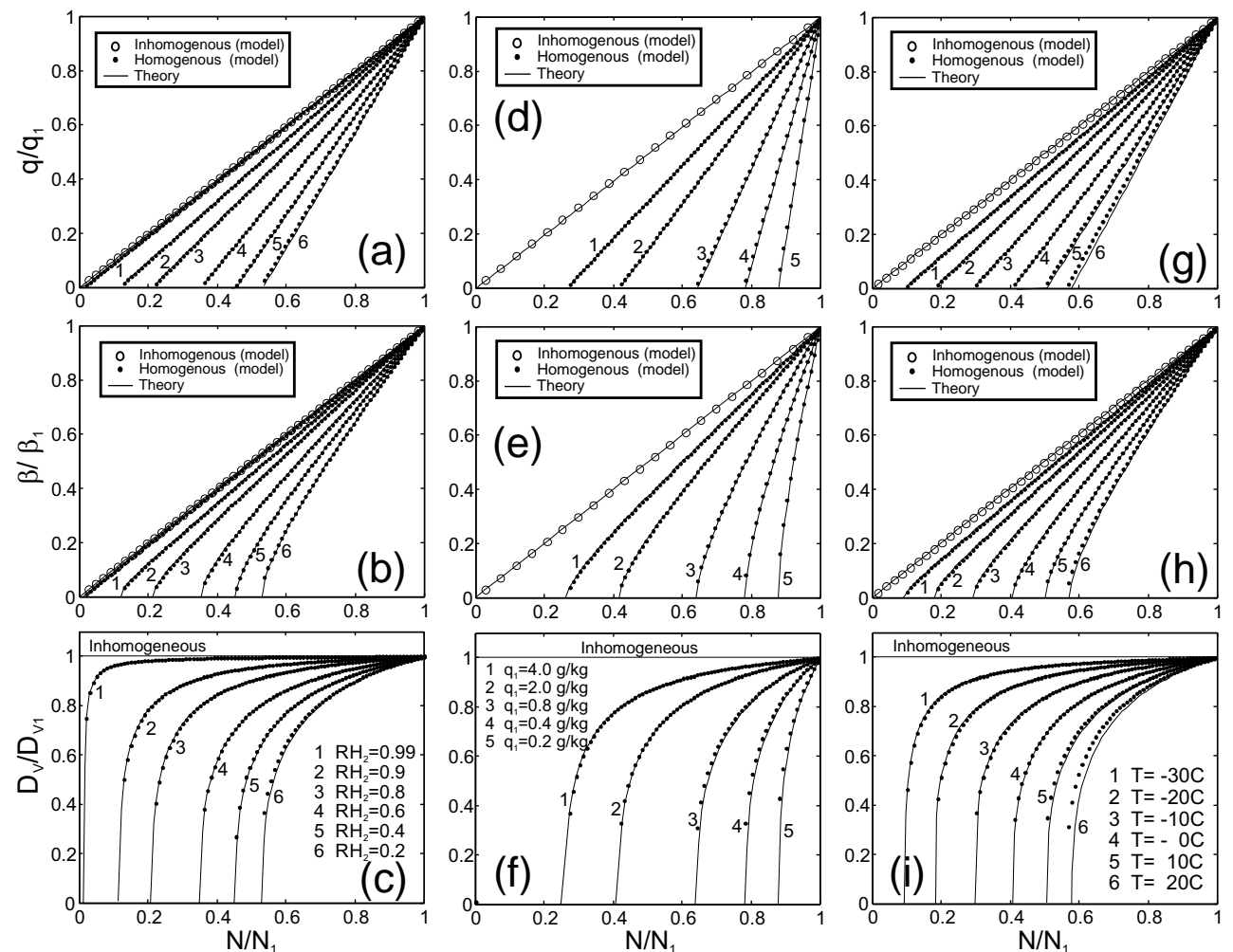

Figure 5. Dependence of normalized liquid water mixing ratio $q / q_{1}(\mathbf{a}, \mathbf{d}, \mathbf{g})$, extinction coefficient $\beta / \beta_{1}(\mathbf{b}, \mathbf{e}, \mathbf{h})$ and mean volume diameter $D_{\mathrm{v}} / D_{\mathrm{v} 1}(\mathbf{c}, \mathbf{f}, \mathbf{i})$ vs. normalized number concentration $N / N_{1}$ for various humidity of the entrained air $(\mathbf{a}, \mathbf{b}, \mathbf{c})$, for various liquid water mixing ratios $(\mathbf{d}, \mathbf{e}, \mathbf{f})$ and for various temperatures $(\mathbf{g}, \mathbf{h}, \mathbf{i})$. The calculations were performed for the following initial conditions: $H=1000 \mathrm{~m}$, $D_{1}=20 \mu \mathrm{m}$; for $(\mathbf{a}-\mathbf{c}) T_{1}=T_{2}=0^{\circ} \mathrm{C}, N_{1}=500 \mathrm{~cm}^{-3} ;(\mathbf{d}-\mathbf{f}) T_{1}=T_{2}=0{ }^{\circ} \mathrm{C}, \mathrm{RH}_{2}=0.5 ;(\mathbf{g}-\mathbf{i}) T_{1}=T_{2}=T, N_{1}=500 \mathrm{~cm}^{-3}, \mathrm{RH}_{2}=0.5$.

cate that the difference between the moments becomes most pronounced at warm temperatures, whereas at cold temperatures (e.g., $\left.T=-30^{\circ} \mathrm{C}\right), q(N), \beta(N)$ and $D_{\mathrm{v}}(N)$ for homogeneous mixing are approaching those for the extreme inhomogeneous mixing limit.

Such behavior is explained by the fact that liquid water deficit $\delta q_{\mathrm{m}}$ decreases with decreasing temperature (Appendix A, Fig. A1). At low temperatures $\left(T=-30^{\circ} \mathrm{C}\right)$ the amount of evaporated water $\delta q_{\mathrm{m}}$ is so small that homogeneous mixing with dry out-of-cloud air will have approximately the same effect as mixing with saturated air (i.e., degenerate case, Sect. 2.7).

Overall, as follows from Fig. 5 the results from the analytical predictions (Sect. 2) turned out to be in a good agreement with numerical simulations.

\subsection{Effect of temperature, case $T_{1} \neq T_{2}$}

Isobaric mixing of two nearly saturated volumes with $T_{1} \neq$ $T_{2}$ may result in supersaturated environment (e.g., Rogers, 1976; Bohren and Albrecht, 1998). Mixing resulting in supersaturation is different in principle from the mixing with evaporating droplets. In this case the meaning of homogeneous and inhomogeneous mixing becomes ambiguous. For- mation of supersaturation leads to different dependences between $N \bar{D}, \beta, q, \bar{D}$ and $N$ as compared to those shown in Figs. 4-5, when $T_{1}=T_{2}$.

Figure 6 presents a set of diagrams similar to those in Fig. 4, but calculated for the cases, when $T_{1} \leq T_{2}$. It turns out that for the case of extreme inhomogeneous mixing the temperature difference between $T_{1}$ and $T_{2}$ breaks down linear dependences of the microphysical moments (e.g., $N \bar{D}$, $\beta, q$ Fig. 6a, c, d) vs. $\mu$.

Figure 7 presents the effect of the temperature difference $\Delta T$ on the normalized dependences $q(N), \beta(N)$ and $D_{\mathrm{v}}(N)$. In clouds, high supersaturation resulting from isobaric mixing may lead to activation of interstitial CCN, which may increase $N$ and decrease $D_{\mathrm{v}}$ (Korolev and Isaac, 2000). However, no activation of new droplets during isobaric mixing was allowed in this study. For the cases when $\mathrm{RH}_{\mathrm{m} 0}>1$ (Fig. 7, AB on line 1) the condensed water was uniformly distributed between available droplets. Therefore, $q(N), \beta(N)$ and $D_{\mathrm{v}}(N)$ calculated for homogeneous and extreme inhomogeneous mixing coincide with each other on this interval.

Numerical simulations also showed that the effect of temperature on mixing is more pronounced for the cases when the cloud temperature is warmer than that of the entrained air, i.e., $T_{1}>T_{2}$, as compared to the cases with $T_{1}<T_{2}$. 

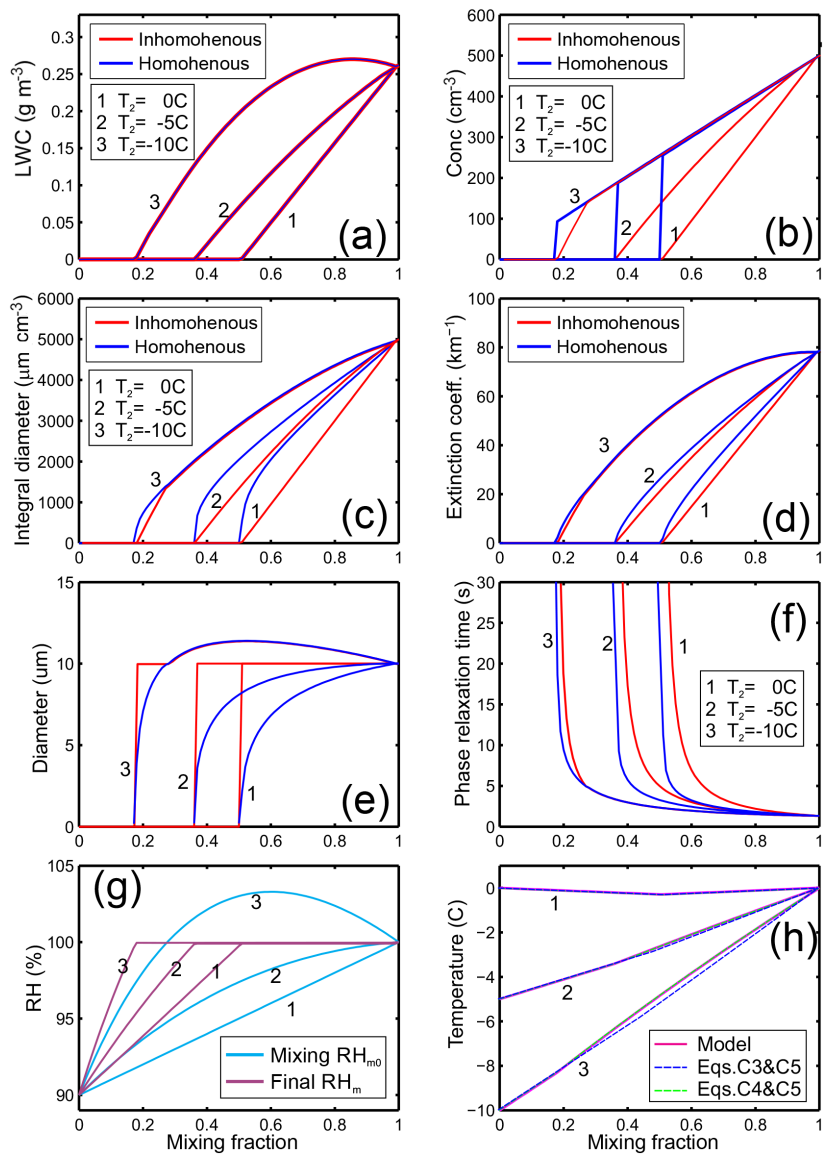

Figure 6. Simulation of (a) droplet number concentration and (b) liquid water mixing ratio, (c) integral droplet diameter, (d) extinction coefficient, (e) mean volume diameter, (f) time of phase relaxation, $(\mathbf{g})$ relative humidity in the mixed volume before droplet evaporation $\mathrm{RH}_{\mathrm{m} 0}$ and at the equilibrium state $\mathrm{RH}_{\mathrm{m}}$, (h) final temperature $T_{\mathrm{m}}$ vs. ratio of mixing $\mu$ formed after homogeneous and extreme inhomogeneous mixing between dry and cloudy parcel with monodisperse droplets. The calculations were performed for $\mathrm{RH}_{2}=0.9 ; D_{1}=10 \mu \mathrm{m}, N_{1}=500 \mathrm{~cm}^{-3} ; T_{1}=0{ }^{\circ} \mathrm{C} ; T_{2}=-10$, $-5,0^{\circ} \mathrm{C} ; H=1000 \mathrm{~m}$.

\section{Progressive mixing}

\subsection{Effect on microphysical parameters}

In the previous sections the mixing was considered as a single event, i.e., $\mu$ fraction of the cloudy air mixed up with $(1-\mu)$ fraction of entrained dry air. Such mixing will be referred to as "primary" mixing. Primary mixing results in an ensemble of elementary volumes characterized by a set of microphysical and state parameters, i.e., $D_{\mathrm{v}}(\mu), N(\mu)$, $\mathrm{RH}(\mu)$ and $T(\mu)$. Each of these parameters has a functional dependence on $\mu$, and what is important, these parameters have functional relationships between each other.

In reality mixing is a continuous process. It does not stop after the primary mixing. The elementary volumes formed
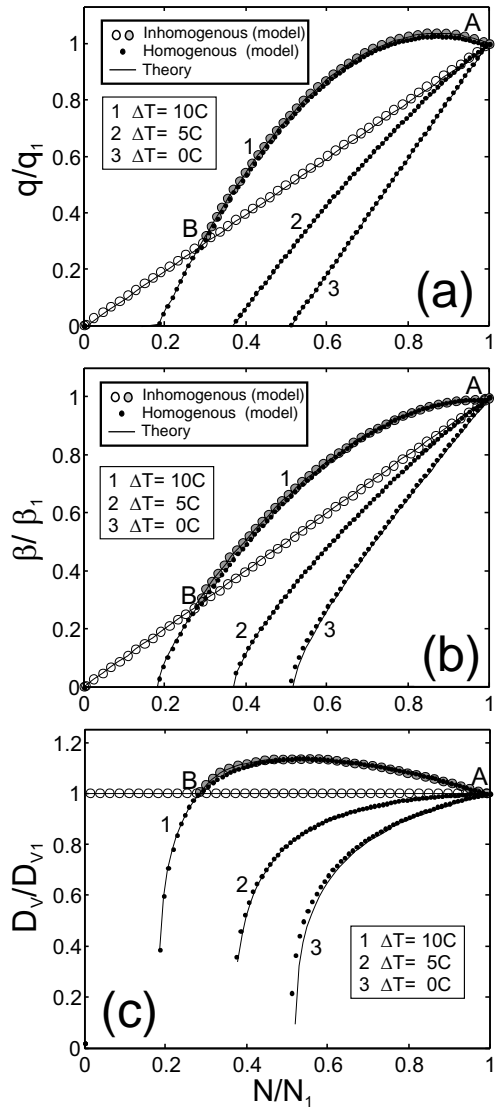

Figure 7. Effect of temperature difference between cloud and entrained air on mixing. The calculations were performed for initial temperatures $T_{2}$ : (1) $-10{ }^{\circ} \mathrm{C}$; (2) $-5{ }^{\circ} \mathrm{C}$; (3) $0{ }^{\circ} \mathrm{C}$. Grey circles indicate extreme inhomogeneous mixing on line 1 at the $\mathrm{AB}$ interval. The rest cases on extreme inhomogeneous mixing are indicated by open circles. The initial conditions used for the calculations were the following: $H=1000 \mathrm{~m}, \mathrm{RH}_{2}=90 \% ; D_{1}=10 \mu \mathrm{m}$, $N_{1}=500 \mathrm{~cm}^{-3}, T_{1}=0{ }^{\circ} \mathrm{C}$.

after primary mixing continue to progressively mix with each other.

The second stage of mixing will result in an ensemble of elementary volumes characterized by a set of parameters $D_{\mathrm{v}}^{(2)}, N^{(2)}, \mathrm{RH}^{(2)}, T^{(2)}$, etc. Here the superscript ${ }^{(2)}$ indicates the stage of mixing. After the second stage the mixed volumes undergo subsequent stages of mixing.

The idealized conceptual diagram of the progressive mixing is shown in Fig. 8. Each stage of mixing results in an ensemble of elementary parcels formed for different $\mu_{j}^{(n)}$. The elementary parcels continue mixing with each other and cloud environment during the subsequent mixing stages. As mentioned in Sect. 2.1, the actual process of mixing is indeed much more complex than the sequence of discrete events portrayed in Fig. 8. However, as it will be shown below, this simplified consideration of progressive mixing allows for the establishment of the main features of evolution of relation- 


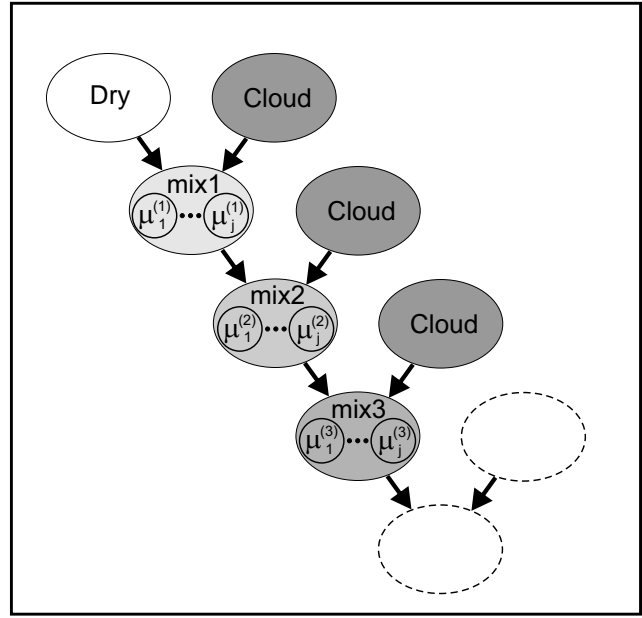

Figure 8. Conceptual diagram of cascade mixing of the out-ofcloud entrained parcel with the cloudy environment.

ships between the microphysical moments affected by mixing. The obtained results facilitate identification of a type of mixing from in situ measurements.

Progressive homogeneous mixing was simulated with the help of a numerical model, where parcels were randomly mixed with each other and with the cloud environment. The mixing fraction $\mu$ was also set to be random during each mixing event. Models of stochastic mixing have been used in a number of studies (e.g., Krueger et al., 1997; Su et al., 1998; Burnet and Brenguier, 2007). In the present work the analysis of progressive mixing is expanded to examine its effect on the relationship between moments of the droplet size distribution.

The results of the progressive homogeneous mixing for the first four stages are presented in Fig. 9. As seen from Fig. 9 the functional relationship between the pairs of microphysical and state parameters exists only for the primary stage. For higher mixing stages these functional relationships break down. Thus, cloud volumes with the same $N^{(2)}$ may have different $D_{\mathrm{v}}^{(2)}$. Figure 9 also shows that the regions of scattering of $q(N), \beta(N)$ and $D_{\mathrm{v}}(N)$ for stages 2, 3 and 4 are limited from above by the inhomogeneous mixing (red dashed lines) and from below by primary homogeneous mixing (red solid lines).

Figure 10 presents a conceptual $N-q$ diagram explaining breaking the functional relationships during progressive homogeneous mixing. After the first stage of mixing the $N-q$ points will be scattered along the line $\mathrm{OB}$ and point $\mathrm{C}$. The line $\mathrm{OB}$ corresponds to the ensemble of points with $\mathrm{RH}=1$. Therefore, the result of mixing between two saturated volumes randomly selected on $\mathrm{AB}$, will remain on the same line. Point $\mathrm{C}$ corresponds to the ensemble of points with $N=$ $0, \mathrm{RH}_{2} \leq \mathrm{RH}_{\mathrm{C}}\left(\mu^{(1)}\right) \leq 1$, where $0 \leq \mu^{(1)}<\mu_{\mathrm{cr}}$. Therefore, mixing between point A (Fig. 10) and point $\mathrm{C}$, when $\mathrm{RH}=1$ will result in scattering along the line $\mathrm{AC}$ (degenerate case).
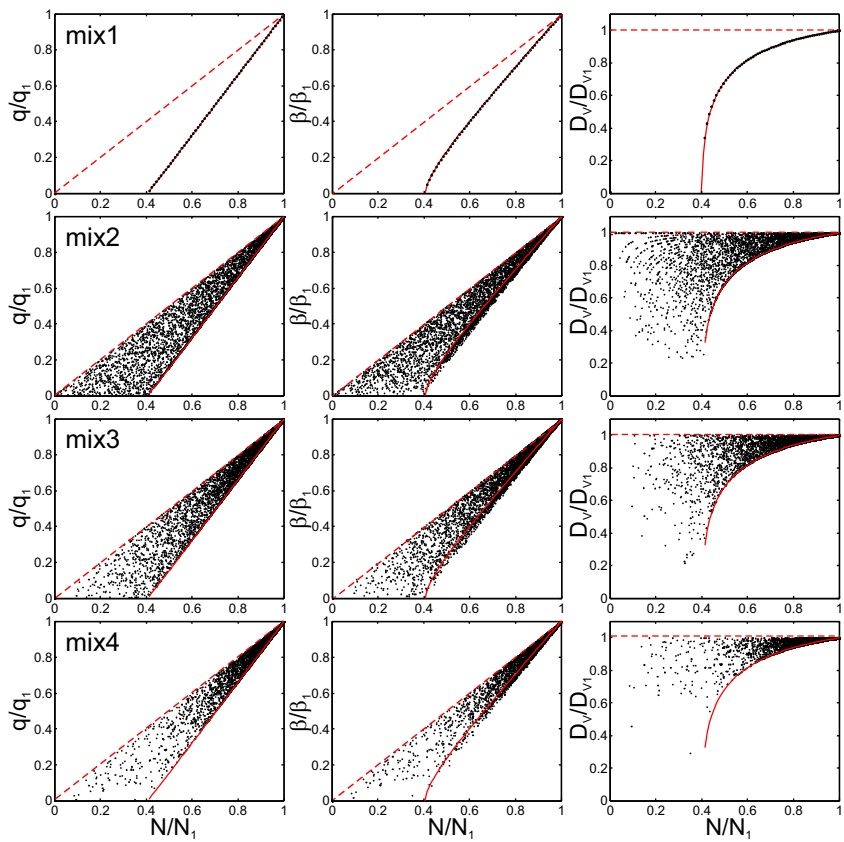

Figure 9. Simulation of stochastic mixing corresponding to stages $1-4$ as indicated in Fig. 8. Solid red lines indicate the normalized dependences $q, \beta, D_{\mathrm{v}}$ vs. $N$ for the primary stage of homogeneous mixing. Dashed red lines indicate the same dependences for inhomogeneous mixing. The initial conditions used for the simulations were the following: $H=1000 \mathrm{~m}, T_{1}=T_{2}=0{ }^{\circ} \mathrm{C} ; \mathrm{RH}_{2}=$ $0.5 ; D_{1}=10 \mu \mathrm{m}, N_{1}=500 \mathrm{~cm}^{-3}$.

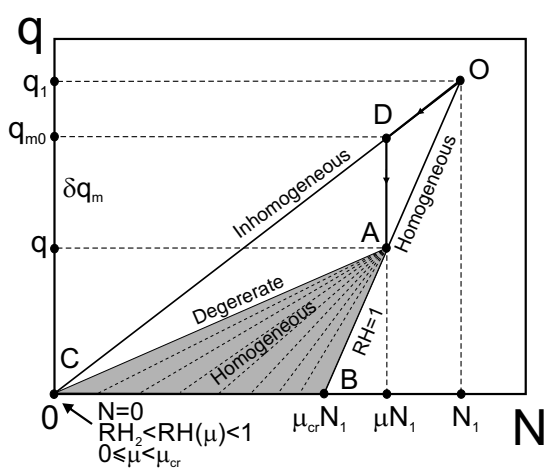

Figure 10. Conceptual diagram explaining breaking the functional relationships between the microphysical moment during progressive missing (see text).

Points resulted from mixing between $\mathrm{A}(\mathrm{RH}=1)$ and point $\mathrm{C}$ when $\mathrm{RH}_{2} \leq \mathrm{RH}_{\mathrm{C}}<1$, will scattered over the ensemble of dashed lines shown in Fig. 10. These lines will fill the sector $\mathrm{CAB}$. Random mixing between points on the line $\mathrm{OB}$ and $\mathrm{C}$ will eventually fill the entire sector COB. The same consideration can be applied to progressive mixing between other moments.

During the progressive mixing $N^{(n)}, \beta^{(n)}, q^{(n)}$ and $D_{\mathrm{v}}^{(n)}$ formed in the elementary parcels tend to approach those in 
the undiluted cloud, i.e., $N_{1}, \beta_{1}, q_{1}$ and $D_{\mathrm{v} 1}$. This process can be considered as a surrogate to the diffusion process between the cloud and sub-saturated out-of-cloud environment. The convergence of $\beta^{(n)}, q^{(n)}$ and $D_{\mathrm{v}}^{(n)}$ during the progressive mixing can be seen in Fig. 9, where the scattering of normalized $q^{(n)}(N), \beta^{(n)}(N)$ and $D_{\mathrm{v}}^{(n)}(N)$ becomes denser towards the top-right corner $(1,1)$ with the increase of the stage of mixing.

It is worth noting that progressive mixing with the dry air does not break the functional relationships between the moments. This case is equivalent to detrainment of cloudy environment into dry air. It can be shown that Eq. (14) remain valid at any stage of progressive homogeneous mixing with dry air only, i.e., $N_{j} / N_{1}=\mu^{(1)} \cdots \mu^{(n-1)} \mu^{(n)}$, where $\mu^{(n)}$ is the mixing fraction at the $n$th stage of mixing. Equations (15)-(18) also remains valid for the progressive mixing with the dry air only.

As follows from Eq. (8) for the case of extreme inhomogeneous mixing the progressive mixing does not affect the functional relations between $N^{(n)}, \beta^{(n)}, q^{(n)}$ and $D_{\mathrm{v}}^{(n)}$ and other microphysical parameters. These relations remain the same regardless of the actual stage of mixing. This is one of the fundamental differences between homogeneous and extreme inhomogeneous mixing, which can be used for identification of type of mixing from in situ measurements.

\subsection{Effect on droplet size distributions}

Figure 11 shows modeled droplet size distributions averaged over the ensembles of elementary volumes corresponding to the first four stages of homogeneous mixing. As seen from Fig. 11a-d for the case with $T_{1}=T_{2}$ the droplet size distributions are broadened towards small sizes. Depending on the stage of mixing and mixing fraction $\mu$ the size distributions formed in each elementary volume may be unimodal or multimodal. However, due to the random nature of the modal sizes formed during mixing, the average size distributions become smooth and unimodal (Fig. 11a-d).

Broadening of droplet size distributions towards small sizes during homogeneous mixing is well known and it was demonstrated in a number of studies (e.g., Baker and Latham, 1982; Jensen and Baker, 1989; Jeffery, 2007; Kumar et al., 2013). However, if mixing results in supersaturation (Sect. 3.4), then the droplet size distribution may broaden towards larger sizes (Fig. 11e-h). For this to occur, both the temperature difference between the cloud and the environment $\left|T_{1}-T_{2}\right|$ and the relative humidity of the environment $\mathrm{RH}_{2}$ must be sufficiently large. Such conditions are inherently unstable, however, this might occur in regions that have been moistened through prior cloud detrainment. Thus homogeneous mixing may result in broadening of droplet size distributions towards either smaller or larger sizes (Fig. 11).

These results were obtained in the frame of the formalism of homogeneous and inhomogeneous mixing. The following two works in this series (Pinsky et al., 2016a, b) will discuss
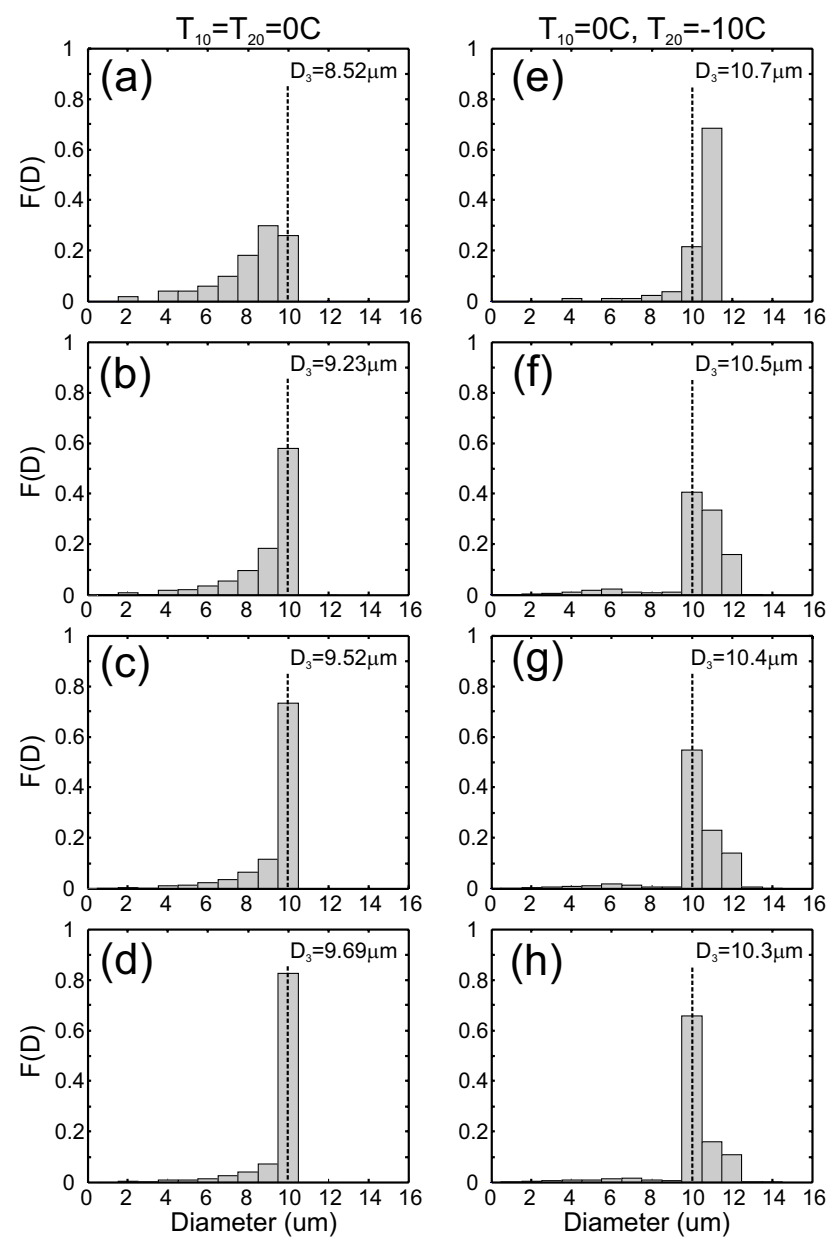

Figure 11. Droplet size distributions formed during the progressive homogeneous mixing corresponding to the (a, e) primary stage; (b, f) 2nd stage; (c, g) 3rd stage; (d, h) 4th stage. Left column (a, b, c, d) corresponds to the case when the cloud temperature is equal to the dry air temperature $T_{1}=T_{2}=0^{\circ}$; right column $(\mathbf{e}, \mathbf{f}, \mathbf{g}, \mathbf{h})$ corresponds to the case when $T_{1}=0^{\circ}, T_{2}=-10^{\circ}$. For both cases the simulation was performed for $D_{1}=10 \mu \mathrm{m} ; N=500 \mathrm{~cm}^{-3}$; $\mathrm{RH}_{2}=0.9$.

the broadening of polydisperse and monodisperse $f(D)$ during both homogeneous and inhomogeneous mixing in greater detail.

\section{Identification of type of mixing from in situ observations}

The purpose of this section is to attempt to identify the type of mixing based on examining relationships between basic microphysical parameters $N, \beta$, LWC, $D_{\mathrm{v}}$ obtained from in situ. 


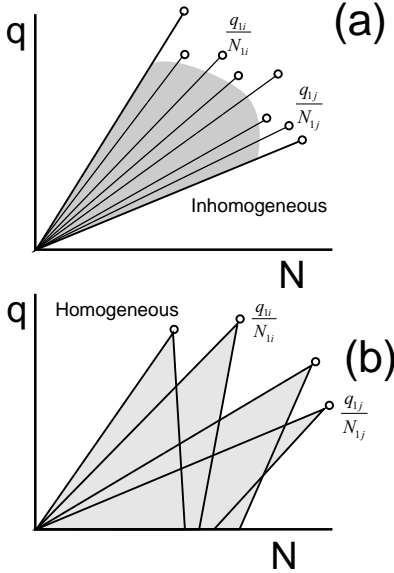

Figure 12. Conceptual diagrams of scattering of measurements of $q$ vs. $N$ for (a) extreme inhomogeneous and (b) homogeneous mixing.

\subsection{Expected relationships between the moments}

Prior to proceeding with the analysis of in situ data we summarize the results of the previous consideration on how homogeneous and extreme inhomogeneous mixing is expected to manifest itself in relationships between basic microphysical parameters, such as $N, \beta, q$ and $D_{\mathrm{v}}$.

For extreme inhomogeneous mixing the relationship between the pairs of $N, \beta$ and $q$ are determined by linear dependencies $M_{n}=\alpha_{n m} M_{m}$ (Eq. 10) at any stage of mixing. As follows from Eq. (11) the slopes $\alpha_{n m}$ for $q(N), \beta(N)$ and $q(\beta)$ are equal to the ratios $q_{1} / N_{1}, \beta_{1} / N_{1}$, and $q_{1} / \beta_{1}$, respectively, where $N_{1}, \beta_{1}$ and $q_{1}$ correspond to undiluted adiabatic values. The values of $N_{1}, \beta_{1}$ and $q_{1}$ may vary depending on the location inside the cloud and environmental conditions at the cloud base. Thus, the adiabatic value of $q_{1}$ is a function of elevation above the cloud base $\Delta Z$, whereas $N_{1}$ depends on the vertical velocity at the cloud base $u_{z}$ and the aerosol load. Therefore, the scattering of $q-N$ points will be aligned along an ensemble of different lines determined by $q_{1} / N_{1}$, which are specific to different cloud volumes. The conceptual diagram of the scattering of $q-N$ measurements in a cloud with extreme inhomogeneous mixing is shown in Fig. 12a. The scatter diagrams for other moments (e.g., $q-\beta$, $N-\beta$ ) will have similar patterns as that in Fig. 12a.

For the case of homogeneous mixing the functional relationship between the pairs of $N, \beta, q$ and $D_{\mathrm{v}}$ are disrupted by a progressive mixing. As shown in Sect. 4.1 the ensemble of points of $N, \beta$ and $q$ will be scattered within a sector, which is limited by lines determined by Eq. (11) (extreme inhomogeneous mixing) and Eqs. (15)-(17) (primary homogeneous), respectively (Fig. 9). What is important is that the top of the sectors for $q(N)$ and $\beta(N)$ correspond to adiabatic points $\left[N_{1}, q_{1}\right]$ and $\left[N_{1}, \beta_{1}\right]$, respectively. Since $N_{1}, \beta_{1}$ and $q_{1}$ may vary within the same cloud, it is anticipated that the
$N, \beta$ and $q$ measurements will be scattered within an ensemble of sectors as shown in Fig. 12b.

It is important to note that during the first stage of homogeneous mixing prior to reaching equilibrium, functional relationships between the microphysical moments do not exist either. After the instant mixing of cloud fraction $\mu$ with entrained air (Fig. 1b2), $q_{\mathrm{m} 0}=\mu q_{0}$ and $N_{\mathrm{m} 0}=\mu N_{0}$. This state corresponds to point D in Fig. 10. After that droplets start evaporating until liquid mixing ratio reaches point A (Fig. 10), which corresponds to the equilibrium state $(\mathrm{RH}=1)$. Therefore, during evaporation time $q-N$ points will be scattered along the line AD. Since, point D can be located anywhere on OC, the ensemble of $q-N$ points corresponding to non-equilibrium state will fill the $\mathrm{COB}$ area.

Thus, the absence of the functional relationships between the moments during homogeneous mixing may occur both during progressive mixing and during primary mixing prior to reaching the equilibrium state. The evaporation time required to reach equilibrium during homogeneous mixing is discussed in detail in Pinsky et al. (2016b), and it is usually limited by a few tens of seconds. However, progressive mixing is not limited in time. Therefore, it is very likely that no functional relationship between microphysical parameters will be observed within a set of in situ measurements.

Figure 12 demonstrated a fundamental difference in scattering of $q-N$ for homogeneous and extreme inhomogeneous mixing, which will be used to facilitate identification of type of mixing in the following section.

\subsection{Results of observations}

The measurements were obtained on the University of Wyoming King Air aircraft during the COPE-MED project in South-Western part of the UK during July-August 2013 (Leon et al., 2016). The UW King Air was equipped with a suite of microphysical instruments, including a DMT Cloud Droplet probe (CDP), designed for measurements of droplet sizes and their concentrations in the nominal size ranges 1$50 \mu \mathrm{m}$.

Figure 13 shows a time series of droplet concentration, extinction coefficient, liquid water content (LWC) and mean volume droplet diameter measured by the CDP during transit through a convective cell on 18 July 2013. The CDP data were sampled at $10 \mathrm{~Hz}$, which corresponds to approximately $10 \mathrm{~m}$ spatial averaging. Visual examination of the spatial changes of $N, \beta$ and LWC shows strong correlation. The amplitude of changes of these parameters reaches nearly 100 percent with respect to their maximum. Contrary to that, the spatial variations of $\bar{D}$ and $D_{\mathrm{v}}$ are quite conservative and their values remain nearly constant. With the exception of two cloud holes between 13:50:42 and 13:50:44, the amplitude of fluctuations of $D_{\mathrm{v}}$ does not exceed $8 \%$ with standard deviation of $2.2 \%$.

Figure 14 shows scatter diagrams of $\operatorname{LWC}(N), \beta(N)$, LWC $(\beta)$ and $D_{\mathrm{v}}(N)$ measured by the CDP during seven con- 


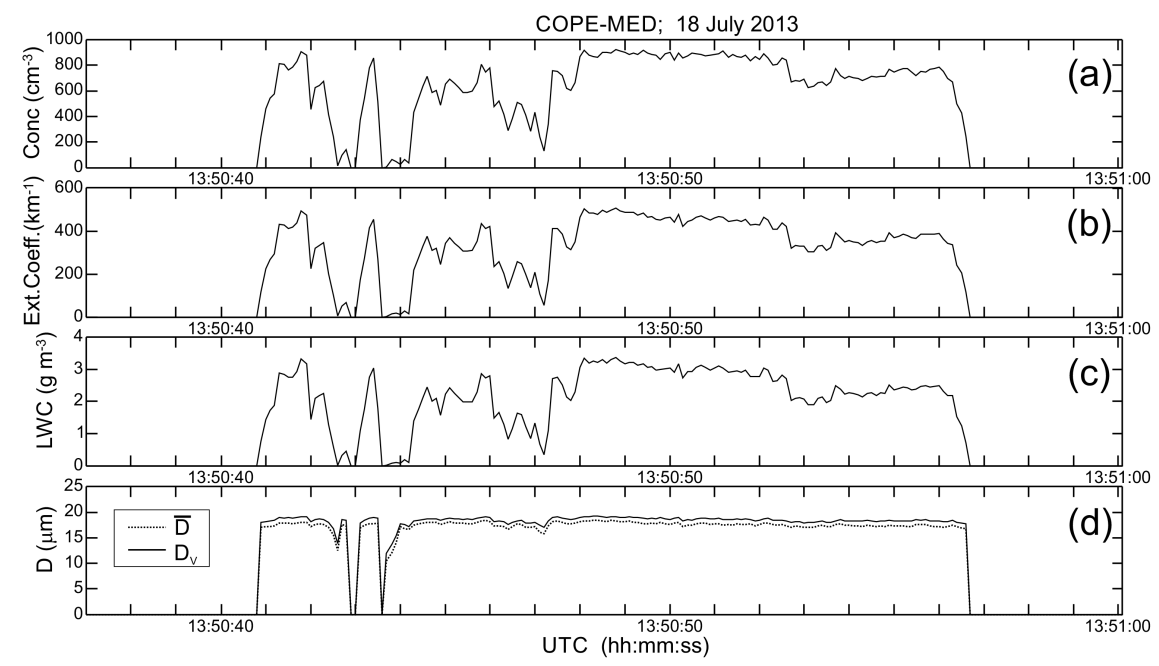

Figure 13. Spatial changes of particle concentration (a), extinction coefficient (b), liquid water content (c) and average and mean mass diameter (d) during transit through one of the convective clouds measured by CDP. The measurements were conducted during the COPEMED project on $18 \mathrm{July} 2015$. The sampling rate $10 \mathrm{~Hz}\left(\sim 10 \mathrm{~m}\right.$ spatial resolution). $H=5500 \mathrm{~m}, T=-12^{\circ} \mathrm{C}, \mathrm{RH}=0.2$.

secutive penetrations of the same convective cell extended over a period of approximately $19 \mathrm{~min}$. One of these penetrations is shown in Fig. 13. The measurements were conducted at $H=5500 \mathrm{~m}$ and $T=-12^{\circ} \mathrm{C}$. The relative humidity of the ambient air was approximately $20 \%$. At the beginning of the sampling no precipitation size particles were observed in the cloud. However, by the end of the sampling period some raindrops and ice crystals were present in the cloud. Despite the presence of some precipitation size particles, the scatter diagrams in Fig. 14a, b and d demonstrate high correlation between pairs $N, \beta$ and LWC. The mean volume diameter in Fig. $14 \mathrm{c}$ shows very little changes from 19 to $17 \mu \mathrm{m}$, when concentration changes from 1100 to $500 \mathrm{~cm}^{-3}$. However, for $N<200 \mathrm{~cm}^{-3}$, the volume diameter decreases to $12-15 \mu \mathrm{m}$.

Red lines in Fig. 14 indicate $q(N), \beta(N), \operatorname{LWC}(\beta)$ and $D_{\mathrm{v}}(N)$ calculated for the 1st stage of homogeneous mixing. The calculations were performed for a monodisperse $f(D)$ with $D_{1}=18.5 \mu \mathrm{m}, N_{1}=1100 \mathrm{~cm}^{-3}$, and state parameters as during the measurements. Comparisons of dependences $q(N), \beta(N), \operatorname{LWC}(\beta)$ and $D_{\mathrm{v}}(N)$ based on in situ measurements with those obtained from numerical simulations of homogeneous mixing show minor difference for high concentrations $700 \mathrm{~cm}^{-3}<N<1100 \mathrm{~cm}^{-3}$ (Fig. 14a-c). The simulation also shows that for this specific case the differences between homogeneous and inhomogeneous mixing does not exceed $10 \%$ when $700 \mathrm{~cm}^{-3}<N<1100 \mathrm{~cm}^{-3}$. Such difference remains within the errors of measurements. Therefore, in this specific cloud for the regions with $N>700 \mathrm{~cm}^{-3}$ the type of mixing cannot be unambiguously identified from the analysis of the dependences $\operatorname{LWC}(N), \beta(N), \operatorname{LWC}(\beta)$ and $D_{\mathrm{v}}(N)$. This is consistent with the assessment of feasibility of segregation of homogeneous and inhomogeneous mixing in Fig. 3 (dashed line). Since for homogeneous mix-
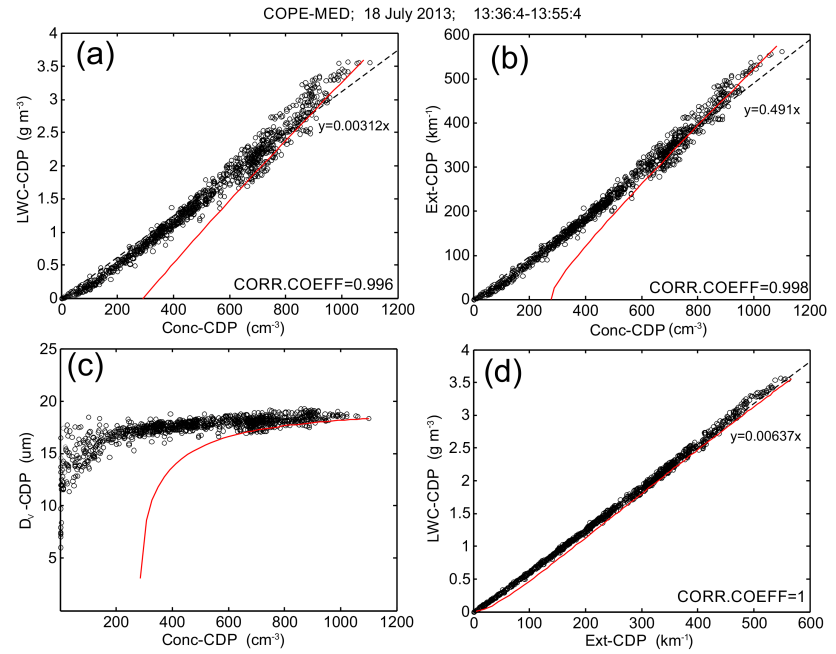

Figure 14. Relationships between (a) $\operatorname{LWC}(N)$; (b) $\beta(N)$; (c) $D_{\mathrm{V}}(N)$; (d) $\operatorname{LWC}(\beta)$ calculated from the CDP measurements obtained during sampling several convective clouds. The measurements were conducted during the COPE-MED project on 18 July $2015, H=5500 \mathrm{~m}, T=-12^{\circ} \mathrm{C}, \mathrm{RH}=0.2$. The measurements were sampled at $10 \mathrm{~Hz}(\sim 10 \mathrm{~m}$ spatial resolution). Dashed lines are linear regressions. Red lines indicate primary inhomogeneous mixing dependencies calculated for the same environmental conditions.

ing $\mu=\frac{N}{N_{1}}$ (Eq. 14), then assuming $N_{1}=1100 \mathrm{~cm}^{-3}$, Fig. 3 suggests good separation of the moments for $N<700 \mathrm{~cm}^{-3}$.

For the regions with $N<500 \mathrm{~cm}^{-3}$ the deviation between homogeneous mixing simulations and in situ measurements in Fig. 14 becomes well pronounced, and it extends beyond possible errors of measurements. This suggests that the mix- 

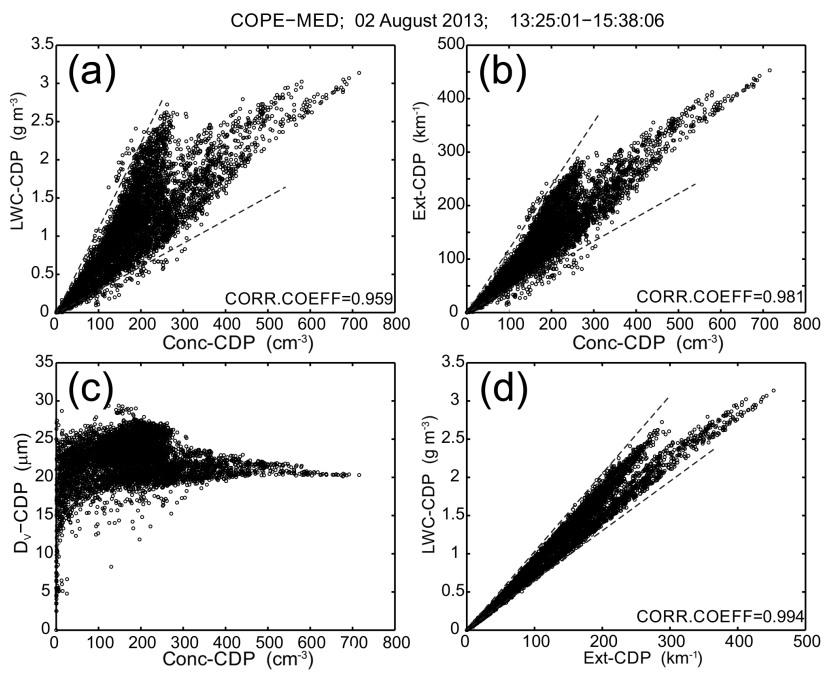

Figure 15. Relationships between (a) $\operatorname{LWC}(N)$; (b) $\beta(N)$; (c) $D_{\mathrm{V}}(N)$; (d) $\mathrm{LWC}(\beta)$ calculated from the CDP measurements sampled during traverse through 45 convective clouds. The measurements were conducted during the COPE-MED project on 2 August 2015. Dashed lines in (a, b, d) indicate the sectors, where the majority of the points are scattered. The altitude of sampling varied in the range $3000 \mathrm{~m}<H<4500 \mathrm{~m}$, temperature $-11^{\circ} \mathrm{C}<T<0{ }^{\circ} \mathrm{C}$, relative humidity in the vicinity of clouds $15 \%<\mathrm{RH}<65 \%$. The measurements were sampled at $10 \mathrm{~Hz}$ $(\sim 10 \mathrm{~m}$ spatial resolution).

ing in these regions is dominated by the extreme inhomogeneous type.

Figure 15 shows the same type of diagrams as in Fig. 14, which were measured during 45 consecutive traverses through an ensemble of deep convective cells. The sampling altitude varied in the range $3000 \mathrm{~m}<H<4500 \mathrm{~m}$, temperature $-11^{\circ} \mathrm{C}<T<0^{\circ} \mathrm{C}$, relative humidity in the vicinity of clouds $15 \%<\mathrm{RH}<65 \%$. The cloud measurements were extended over a period of $2 \mathrm{~h} 13 \mathrm{~min}$, which is suggestive that the convective cells were sampled at different stages of their lifetime. At the sampling level the concentration of raindrops varied from zero to a few per liter, and their diameter did not exceed $2 \mathrm{~mm}$.

What is interesting is that the scattering of $\operatorname{LWC}(N), \beta(N)$ and $\operatorname{LWC}(\beta)$ (Fig. 15a, b and d) is limited by the sector, which originates from the zero point as in Fig. 12a. Analysis of the measurements showed that the data points $\operatorname{LWC}(N)$, $\beta(N), \operatorname{LWC}(\beta)$ in each individual cloud traverse are well aligned along the lines with different slopes (e.g., Fig. 14). After averaging over the ensemble of clouds, the area of the scattered points turned out to be located inside a sector limited by the lines with smallest and largest slopes.

Comparisons of the scatter diagrams $\operatorname{LWC}(N), \beta(N)$ and $\operatorname{LWC}(\beta)$ in Figs. 14 and 15 with the conceptual diagrams in Fig. 12 unambiguously suggest that interaction between cloud and environment in the studied clouds was dominated by inhomogeneous mixing. It should be emphasized that analysis of a stand-alone mixing diagram $N-D_{\mathrm{v}}$ would not allow one to unambiguously draw such a conclusion.

\section{Discussion}

One of the assumptions in most past studies is that for a sequence of the cloud samples collected along the flight path, the adiabatic values of $N_{1}, q_{1}, \beta_{1}, D_{1}$ and environmental parameters $e_{2}$ and $T_{2}$ remain the same. In fact these parameters may vary both within the same cloud or sequence of sampled clouds, and the amplitude of their variations depends on microphysical and thermodynamical properties inside and outside the cloud environment. This variation will result in an ensemble of relationships $M_{n}=F_{n k}\left(M_{k}\right)$, and enhance scattering of the data points. In such cases identification of the type of mixing based on the $N-D_{\mathrm{v}}$ diagram may result in confusion between homogeneous and inhomogeneous mixing. As demonstrated in Sect. 5, consideration of $N-q$ and $N-\beta$ diagrams may provide a better identification of type of mixing.

Strictly speaking the identification of type of mixing from particle probe measurements as it was performed in Sect. 5 is incomplete. It allows for the establishment of a correlation between microphysical moments and makes a formal conclusion about the mixing type, however it does not allow judgement about stage of mixing (i.e., whether mixing is complete by reaching equilibrium). In most previous studies, and also this one, identification of type of mixing was based on the assumption that the sampled cloud volume is in equilibrium state $(\mathrm{RH}=1)$, and that it reached the final stage of mixing (Fig. 1a2, a3, b3). It is possible that at the moment of measurement the process of mixing is not complete and the droplet-free filaments remained undersaturated (Fig. 1a1, b1, b2). In this case the relationship between different moments may be well described as $M_{n}=\alpha_{n k} M_{n k}$ and the mixing may be confused with inhomogeneous mixing.

In order to identify stage of mixing, high-frequency collocated measurements of temperature and humidity are required. Unfortunately, current technology does not allow such measurements yet.

The basic assumption underlying the analysis of relationships between moments is that the cloud environment is not affected by other non-adiabatic processes. Thus, collisioncoalescence, riming or Wegener-Bergeron-Findeisen processes may change the droplet number concentration and liquid water content, and therefore, affect the relationship between the moments. Activation of interstitial $\mathrm{CCN}$ will result in breaking correlation between the moments due to formation of large concentration of droplets. Broad size distributions may also hinder identification of type of mixing due to partial evaporation of small droplets (Pinsky et al., 2016a).

It is anticipated that most suitable candidates to study mixing-entrainment processes are non-precipitating convec- 
tive clouds and stratocumulus clouds with relatively narrow droplet size distributions.

Another limiting factor is that the above consideration did not account for the effect of changing relative humidity in a vertically ascending parcel. Thus in droplet free entrained air RH increases approximately $10 \%$ for $\Delta z=200 \mathrm{~m}$ at $T=0{ }^{\circ} \mathrm{C}$. After reaching saturation the mixing turns into a degenerate case, which will appear as extreme inhomogeneous mixing. Joint effects of evaporating droplets and an increase in RH during the vertical ascent may facilitate reaching saturation state. This case may also be relevant to the convective cloud described in Sect. 5.2.

\section{Conclusions}

This study analyzes dependences of different moments of $f(D)$ in the frame of formalism of homogeneous and extreme inhomogeneous mixing. The analysis was performed for the final stage of mixing based on the mass and energy conservation consideration. The following results were obtained in the frame of this study:

1. Simple analytical relationships between the main microphysical moments were obtained for the final state homogenous and extreme inhomogeneous mixing.

2. It was shown that the functional relationships between the moments exist only for the first stage of homogeneous mixing when equilibrium is reached. Subsequent progressive homogeneous mixing breaks the functional relationship between the moments.
3. It was demonstrated that consideration of scattering $N-$ LWC, $N-\beta$ diagrams facilitates identification of type of mixing from in situ measurements. For extreme inhomogeneous mixing the scattering of the data points $N-$ LWC, $N-\beta$ will be limited by a sector originating at zero point (Fig. 12a). However, for homogeneous mixing the scattering data points will be limited by a sector originating at $\left(N_{1} \mathrm{LWC}_{1}\right)$ and $\left(N_{1} \beta_{1}\right)$ (Fig. 12b). Utilizing a stand-alone conventional $N-D_{\mathrm{v}}$ mixing diagram may not provide unambiguous answers about type of mixing.

4. The developed approach was applied to a set of in situ measurements collected in convective clouds. The analysis of the dependences between $N, \beta, \mathrm{LWC}$ and $D_{\mathrm{v}}$ suggests that the interaction between entrained and cloudy environments for the studied clouds was dominated by inhomogeneous mixing.

The present study considers relationships between different moments of $f(D)$ for the final stage of mixing. The following two works by Pinsky et al. (2016a, b) in this series provide a detailed analysis of time dependences of droplet size distributions and its moments during homogeneous and inhomogeneous mixing.

\section{Data availability}

The UW King Air data from the COPE-MED project, which were used in this study, are published at University of Wyoming, Research Flight Center (2016). 


\section{Appendix A: Liquid water deficit}

The objective of this section is to find the amount of liquid water, which is required to be evaporated in order to saturate the parcel formed after mixing. Assume that $q_{\mathrm{v} 1}, q_{\mathrm{v} 2}$ are the mixing vapor ratios in the cloudy and entrained parcels, respectively, and $T_{1}, T_{2}$ are their respective initial temperatures. First, we find the saturation ratio $S_{\mathrm{m} 0}$ formed after instant mixing of the cloud and entrained before the cloud droplets start evaporating.

The vapor mixing ratio $q_{\mathrm{vm}}$ formed in the mixed volume will be

$q_{\mathrm{vm}}=\mu q_{\mathrm{v} 1}+(1-\mu) q_{\mathrm{v} 2}$.

The vapor pressure $e_{\mathrm{m}}$ in the mixed volume can be derived from Eq. (A1) by substituting $q_{\mathrm{v}}=\frac{e}{p-e} \frac{R_{\mathrm{a}}}{R_{\mathrm{v}}}$, i.e.,

$e_{\mathrm{m}}=p \frac{\mu+\frac{e_{2}\left(p-e_{1}\right)}{p\left(e_{1}-e_{2}\right)}}{\mu+\frac{\left(p-e_{1}\right)}{\left(e_{1}-e_{2}\right)}}$.

The temperature of the mixed volume $T_{\mathrm{m} 0}$ can be found from the energy conservation law

$$
\begin{aligned}
& \mu\left(q_{\mathrm{v} 1} c_{p \mathrm{v}}+c_{p \mathrm{a}}\right)\left(T_{1}-T_{\mathrm{m} 0}\right) \\
& \quad=(1-\mu)\left(q_{\mathrm{v} 2} c_{p \mathrm{v}}+c_{p \mathrm{a}}\right)\left(T_{\mathrm{m} 0}-T_{2}\right),
\end{aligned}
$$

here $c_{p \mathrm{v}}, c_{p \mathrm{a}}$, are the specific heat capacitance of water vapor and dry air at constant pressure, respectively. Substituting $q_{\mathrm{v} 1}, q_{\mathrm{v} 2}$ yields the temperature in the mixed volume

$T_{\mathrm{m} 0}=\frac{\mu T_{1}+\alpha(1-\mu) T_{2}}{\mu+\alpha(1-\mu)}$

here

$\alpha=\frac{1+\frac{c_{p \mathrm{~V}} R_{\mathrm{a}} e_{2}}{c_{p \mathrm{a}} R_{\mathrm{V}}\left(p-e_{2}\right)}}{1+\frac{c_{p \mathrm{v}} R_{\mathrm{a}} e_{1}}{c_{p \mathrm{a}} R_{\mathrm{V}}\left(p-e_{1}\right)}}$

With a good accuracy $\alpha \cong 1$. The resulting relative humidity after mixing the two volumes will be

$\mathrm{RH}_{\mathrm{m} 0}=\frac{e_{\mathrm{m} 0}}{e_{S}\left(T_{\mathrm{m} 0}\right)}$,

where $e_{s}\left(T_{\mathrm{m} 0}\right)$ is the saturated vapor pressure at temperature $T_{\mathrm{m} 0}$.

The process of evaporation is accompanied by changing humidity and temperature due to latent heat of vaporization. This process is described by the Eq. (C2) in Korolev and Mazin (2003). Assuming the process to be isobaric (i.e., vertical velocity $\left.u_{z}=0\right)$ and the absence of ice $\left(\mathrm{d} q_{i}=0\right)$, Eq. (C2) (Korolev and Mazin, 2003) yields

$$
\frac{\mathrm{d} S}{S+1}=\left(\frac{1}{S+1} \frac{p R_{\mathrm{v}}}{e_{S} R_{\mathrm{a}}}+\frac{L^{2}}{c_{p \mathrm{a}} R_{\mathrm{v}} T^{2}}\right) \mathrm{d} q .
$$

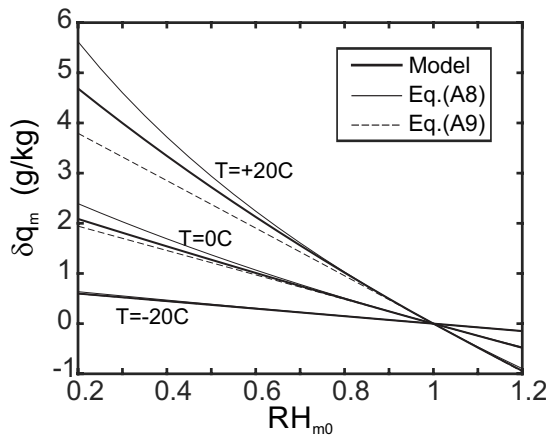

Figure A1. Amount of evaporated liquid water $\delta q_{\mathrm{m}}$ required for saturation of a cloud volume with initial humidity $\mathrm{RH}_{\mathrm{m}}$. Comparisons of the modeled $\delta q_{\mathrm{m}}$ and that calculated from Eqs. (A8) and (A9) for three temperatures $T_{\mathrm{m} 0}=-20,0$ and $20^{\circ} \mathrm{C}$. Calculations were performed for $P=880 \mathrm{mb}$.

Integrating Eq. A7) from initial $S_{\mathrm{m} 0}$ to saturation state, when $S=0$, and taking into account that $\mathrm{RH}=S+1$, gives

$\delta q_{\mathrm{m}}=-b \ln \left(\frac{1+a \mathrm{RH}_{\mathrm{m} 0}}{1+a}\right)$

the mixing ratio of liquid water required to evaporate in order to saturate $1 \mathrm{~kg}$ of the cloud volume formed after mixing with the entrained air, but before droplets start evaporating. Here $a=\frac{e_{s} R_{\mathrm{a}} L^{2}}{p c_{p} R_{\mathrm{v}}^{2} T_{\mathrm{m0}}^{2}}, b=\frac{c_{p} R_{\mathrm{V}} T_{\mathrm{m} 0}^{2}}{L^{2}}$.

Rewriting the right side of Eq. (A8) as $-b \ln \left(1+\frac{a\left(\mathrm{RH}_{\mathrm{m} 0}-1\right)}{1+a}\right)$, and taking into account that $\left|\frac{a\left(\mathrm{RH}_{\mathrm{m} 0}-1\right)}{1+a}\right|<1$, Eq. (A8) can be simplified as

$\delta q_{\mathrm{m}}=a b \frac{1-\mathrm{RH}_{\mathrm{m} 0}}{1+a}=-\frac{S_{\mathrm{m} 0}}{A_{2}}$,

where $A_{2}=\frac{a b}{1+a}$. The analysis of Eqs. (A8)-(A9) shows that for a wide range of temperatures $-30^{\circ} \mathrm{C}<T<30^{\circ} \mathrm{C}$, both equations hold with high accuracy as long as the temperatures of the sub-saturated and cloud parcels $\left|T_{1}-T_{2}\right|<10^{\circ} \mathrm{C}$.

Figure A1 shows comparisons of modeled $\delta q_{\mathrm{m}}$ and that calculated from Eqs. (A8) and (A9) for three different temperatures. The model solved a system of differential equation with incremental evaporation of liquid water until saturation is reached. As seen from Fig. A1 the agreement between modeled $\delta q_{\mathrm{m}}$ and that calculated from Eqs. (A8)-(A9) is quite good and does not exceed few percent at $\mathrm{RH}_{\mathrm{m} 0}=0.5$. This discrepancy results from assumption that $e_{s}$ and $T$ are constant in Eqs. (A8)-(A9). 


\section{Appendix B: Liquid water deficit when $T_{1}=T_{2}$}

Equation (A2) by assuming that $p \gg e_{1}$ and $p \gg e_{2}$ can be simplified as

$e_{\mathrm{m} 0}=\mu e_{1}+(1-\mu) e_{2}$.

As follows from Eq. (A4) for the case $T_{1}=T_{2}$ with high accuracy $T_{\mathrm{m} 0}=T_{1}=T_{2}$. Therefore, $e_{s}\left(T_{\mathrm{m} 0}\right)=e_{s}\left(T_{1}\right)=$ $e_{s}\left(T_{2}\right)$. Dividing Eq. (B1) by $e_{s}$ yields

$\mathrm{RH}_{\mathrm{m} 0}=\mu \mathrm{RH}_{1}+(1-\mu) \mathrm{RH}_{2}$.

In most liquid clouds $\mathrm{RH}_{1}=1$ (Korolev and Mazin, 2003).

Therefore, Eq. (B2) turns into

$\mathrm{RH}_{\mathrm{m} 0}=\mu+(1-\mu) \mathrm{RH}_{2}$.

Substituting Eq. (B3) in Eq. (A8) yields

$\delta q_{\mathrm{m}}=-b \ln \left(1+\frac{a(1-\mu)\left(\mathrm{RH}_{2}-1\right)}{1+a}\right)$.

The expression under logarithm can be presented as the first two terms of the series expansion of $\left(1+\frac{a\left(\mathrm{RH}_{2}-1\right)}{1+a}\right)^{(1-\mu)}$. Substituting this expression into Eq. (B4), gives

$\delta q_{\mathrm{m}}=(1-\mu) \delta q^{*}$,

where

$\delta q^{*}=-b \ln \left(\frac{1+a \mathrm{RH}_{2}}{1+a}\right)$

is the mixing ratio of liquid water required to saturate $1 \mathrm{~kg}$ of the entrained dry volume.

\section{Appendix C: Temperature in the mixing volume}

The energy conservation for evaporating droplets can be written as

$\left(T-T_{\mathrm{m} 0}\right)\left(1+q_{\mathrm{vm}}\right) c_{p \mathrm{~m}}+(1-\mu) \delta q^{*} L=0$,

here $c_{p \mathrm{~m}}$ is the specific heat capacity of the moist air

$c_{p \mathrm{~m}}=\frac{c_{p \mathrm{a}}+q_{\mathrm{vm}} c_{p \mathrm{v}}}{1+q_{\mathrm{vm}}}$.

Since $q_{\mathrm{vm}} \ll 1$ and, $c_{p \mathrm{a}} \cong c_{p \mathrm{~m}}$ Eq. (C1) may be simplified, so that the final temperature after mixing

$T=T_{\mathrm{m} 0}-\frac{(1-\mu) \delta q^{*} L}{c_{p \mathrm{a}}}$.

For the case when $T_{1} \neq T_{2}$ Eq. (C3) should be replaced by

$T=T_{\mathrm{m} 0}-\frac{\delta q_{\mathrm{m}} L}{c_{p \mathrm{a}}}$.

Eqs. (C3) and (C4) are valid for the mixing fraction $\mu>\mu_{\mathrm{cr}}$. For $\mu \leq \mu_{\mathrm{cr}}$ all entrained liquid water $\mu q_{0}$ evaporates, and the final temperature will be

$$
T=T_{\mathrm{m} 0}-\frac{\mu q_{0} L}{c_{p \mathrm{a}}} .
$$


Acknowledgements. The authors appreciate two anonymous reviewers for their comments. Alexei Korolev work was supported by Environment Canada and Transport Canada. The COPE-MED project was funded by National Science Foundation grant AGS1230292 and AGS-1230203. The contribution of Mark Pinsky and Alex Khain was supported by the Israel Science Foundation (grant 1393/14), the Office of Science (BER), US Department of Energy Award DE-SC0006788 and the Binational US-Israel Science foundation (grant 2010446).

Edited by: T. Garrett

Reviewed by: two anonymous referees

\section{References}

Andrejczuk, M., Grabowski, W. W., Malinowski, S. P., and Smolarkiewicz, P. K.: Numerical simulation of cloud-clear air interfacial mixing: homogeneous vs. inhomogeneous mixing, J. Atmos. Sci., 66, 2493-2500, 2009.

Baker, M. B. and Latham, J.: The evolution of droplet spectra and the rate of production of embryonic raindrops in small cumulus clouds, J. Atmos. Sci., 36, 1612-1615, 1979.

Baker, M. B. and Latham, J.: A diffusive model of the turbulent mixing of dry and cloudy air, Q. J. Roy. Meteor. Soc., 108, 871898, 1982.

Baker, M. B., Corbin, R. G., and Latham, J.: The influence of entrainment on the evolution of cloud droplet spectra: I. A model of inhomogeneous mixing, Q. J. Roy. Meteor. Soc., 106, 581-598, 1980.

Beals, M. J., Fugal, J. P., Shaw, R. A., Lu, J., Spuler, S. M., and Stith, J. L.: Holographic measurements of inhomogeneous cloud mixing at the centimeter scale, Science, 350, 87-90, 2015.

Blyth, A. M. and Latham, J.: A Climatological Parameterization for Cumulus Clouds, J. Atmos. Sci., 48, 2367-2371, 1991.

Bohren, C. F. and Albrecht, C. H.: Atmospheric Thermodynamics, Oxford University Press, New York, 402 pp., 1998.

Bower, K. N. and Choularton, T. W.: The effects of entrainment on the growth of droplets in continental cumulus clouds, Q. J. Roy. Meteor. Soc., 114, 1411-1434, 1988.

Broadwell, J. E. and Breidenthal, R. E.: A simple model of mixing and chemical reaction in a turbulent shear layer, J. Fluid Mech., 125, 397-410, 1982.

Burnet, F. and Brenguier, J. L.: Observational study of the entrainment-mixing process in warm convective clouds, J. Atmos. Sci., 64, 1995-2011, 2007.

Devenish, B. J., Bartello, P., Brenguier, J.-L., Collins, L. R., Grabowski, W. W., Ijzermans, R. H. A., Malinovski, S. P., Reeks, M. W., Vassilicos, J. C., Wang, L.-P., and Warhaft, Z.: Droplet growth in warm turbulent clouds, Q. J. Roy. Meteor. Soc., 138, 1401-1429, 2012.

Gerber, H., Frick, G., Jensen, J. B., and Hudson, J. G.: Entrainment, mixing, and microphysics in trade-wind cumulus, J. Meteorol. Soc. Jpn., 86, 87-106, 2008.

Hill, T. A. and Choularton, T. W.: An airbone study of the microphysical structure of cumulus clouds, Q. J. Roy. Meteor. Soc., 111, 517-544, 1985.

Jarecka, D., Grabowski, W. W., Morrison, H., and Pawlowska, H.: Homogeneity of the Subgrid-Scale Turbulent Mixing in Large-
Eddy Simulation of Shallow Convection, J. Atmos. Sci., 70, 2751-2767, 2013.

Jeffery, C. A.: Inhomogeneous cloud evaporation, invariance, and Damköhler number, J. Geophys. Res., 112, D24S21, doi:10.1029/2007JD008789, 2007.

Jensen, J. and Baker, M.: A simple model of droplet spectra evolution during turbulent mixing, J. Atmos. Sci., 46, 2812-2829, 1989.

Korolev, A. V.: The influence of supersaturation fluctuations on droplet spectra formation, J. Atmos. Sci., 52, 3620-3634, 1995.

Korolev, A. V. and Isaac, G. A.: Drop growth due to high supersaturation caused by isobaric mixing, J. Atmos. Sci., 57, 1675-1685, 2000.

Korolev, A. V. and Mazin, I. P.: Supersaturation of water vapor in clouds, J. Atmos. Sci., 60, 2957-2974, 2003.

Krueger, S., Su, C.-W., and McMurtry, P.: Modeling entrainment and finescale mixing in cumulus clouds, J. Atmos. Sci., 54, 2697-2712, 1997.

Kumar, B., Schumacher, J., and Shaw, R. A.: Cloud microphysical effects of turbulent mixing and entrainment, Theor. Comp. Fluid Dyn., 27, 361-376, 2013.

Lasher-Trapp, S. G., Cooper, W. A., and Blyth, A. M.: Broadening of droplet size distributions from entrainment and mixing in a cumulus cloud, Q. J. Roy. Meteor. Soc., 131, 195-220, 2005.

Latham, J. and Reed, R. L.: Laboratory studies of the effects of mixing on the evolution of cloud droplet spectra, Q. J. Roy. Meteor. Soc., 103, 297-306, 1977.

Lehmann, K., Siebert, H., and Shaw, R. A.: Homogeneous and inhomogeneous mixing in cumulus clouds: dependence on local turbulence structure, J. Atmos. Sci., 66, 3641-3659, 2009.

Leon, D. C., French, J. R., Lasher-Trapp, S., Blyth, A. M., Abel, S. J., Ballard, S., Bennett, L. J., Bower, K., Brooks, B., Brown, P., Choularton, T., Clark, P., Collier, C., Crosier, J., Cui, Z., Dufton, D., Eagle, C., Flynn, M. J., Gallagher, M., Hanley, K., Huang, Y., Kitchen, M., Korolev, A., Lean, H., Liu, Z., Marsham, J., Moser, D., Nicol, J., Norton, E. G., Plummer, D. Price, J., Ricketts, H., Roberts, N., Rosenberg, P. D., Taylor, J. W., Williams, P. I., and Young, G.: The Convective Precipitation Experiment (COPE): investigating the origins of heavy precipitation in the southwestern UK, B. Am. Meteorol. Soc., in press, 2016.

Lu, C., Liu, Y., and Niu, S.: Examination of turbulent entrainmentimixing mechanisms using a combined approach, J. Geophys. Res., 116, D20207, doi:10.1029/2011JD015944, 2011.

Lu, C., Liu, Y., Niu, S., and Endo, S.: Scale dependence of entrainment-mixing mechanisms in cumulus clouds, J. Geophys. Res.-Atmos., 119, 13877-13890, doi:10.1002/2014JD022265, 2014.

Paluch, I. R.: Mixing and the droplet size spectrum: generalizations from the CCOPE data, J. Atmos. Sci., 43, 1984-1993, 1986.

Paluch, I. R. and Baumgardner, D. G.: Entrainment and fine-scale mixing in a continental convective cloud, J. Atmos. Sci., 46, 261278, 1989.

Paluch, I. R. and Knight, C. A.: Mixing and evolution of cloud droplet size spectra in a vigorous continental cumulus, J. Atmos. Sci., 41, 1801-1815, 1984.

Pinsky, M., Khain, A., Korolev, A., and Magaritz-Ronen, L.: Theoretical investigation of mixing in warm clouds - Part 2: Homogeneous mixing, Atmos. Chem. Phys., 16, 9255-9273, doi:10.5194/acp-16-9255-2016, 2016a. 
Pinsky, M., Khain, A., and Korolev, A.: Theoretical analysis of mixing in liquid clouds - Part 3: Inhomogeneous mixing, Atmos. Chem. Phys., 16, 9273-9297, doi:10.5194/acp-16-92732016, 2016b.

Rogers, R. R.: A Short Course in Cloud Physics, Pergamon press, Oxford, 227 pp., 1976.

Squires, P.: The growth of cloud drops by condensation, Aust. J. Sci. Res., 5, 66-86, 1952.

Su, C.-W., Krueger, S. K., McMurtry, P. A., and Austin, P. H.: Linear eddy modeling of droplet spectral evolution during entrainment and mixing in cumulus clouds, Atmos. Res., 47-48, 41-58, 1998.
Tolle, M. H. and Krueger, S. K.: Effects of entrainment and mixing on droplet size distributions in warm cumulus clouds, J. Adv. Model. Earth Syst., 6, 281-299, doi:10.1002/2012MS000209, 2014.

University of Wyoming, Research Flight Center: Flight Level Data from the University of Wyoming King Air during the Convective Precipitation Experiment-Microphysics and Entrainment Dependencies (COPE-MED), Version 1.0, doi:10.15786/M2MW2S, 2016. 